\title{
Actin nucleation safeguards single-stranded DNA and promotes replication fork protection
}

Jadwiga Nieminuszczy

Institute of Cancer Research https://orcid.org/0000-0003-4672-9684

Peter Martin

Institute of Cancer Research https://orcid.org/0000-0003-2048-421X

\section{Ronan Broderick}

Institute of Cancer Research

Joanna Krwawicz

Institute of Cancer Research

Alexandra Kanellou

Institute of Cancer Research

\section{Vicky Bousgouni}

Institute of Cancer Research

\section{Chris Bakal}

Institute of Cancer Research https://orcid.org/0000-0002-0413-6744

\section{Beth Woodward}

University of Birmingham Institute of Cancer and Genomic Sciences

\section{Fiona Shackley}

Sheffield Children's Hospital NHS Foundation Trust

\section{Andrés Aguilera}

Andalusian Molecular Biology and Regenerative Medicine Centre https://orcid.org/0000-0003-4782-

1714

\section{Grant Stewart}

University of Birmingham https://orcid.org/0000-0002-0960-3241

\section{Yatin Vyas}

University of lowa Children's Hospital https://orcid.org/0000-0002-4595-0267

Wojciech Niedzwiedz ( $\square$ wojciech.niedzwiedz@icr.ac.uk)

Institute of Cancer Research

\section{Article}

Keywords: DNA replication, single-stranded DNA, genome stability, actin, actin nucleation 
DOl: https://doi.org/10.21203/rs.3.rs-806457/v1

License: (c) (1) This work is licensed under a Creative Commons Attribution 4.0 International License. Read Full License 


\section{Actin nucleation safeguards single-stranded DNA and promotes replication fork protection}

Jadwiga Nieminuszczy $^{1,2}$, Peter R. Martin ${ }^{1,2}$, Ronan Broderick ${ }^{1}$, Joanna Krwawicz ${ }^{1}$, Alexandra Kanellou$^{1}$, Vicky Bousgouni ${ }^{1}$, Chris Bakal $^{1}$, Beth L. Woodward ${ }^{3}$, Fiona Shackley ${ }^{4}$, Andres Aguilera $^{5}$, Grant S. Stewart ${ }^{3}$, Yatin M. Vyas ${ }^{6 *}$ and Wojciech Niedzwiedz ${ }^{1 *}$.

1. Cancer Biology, The Institute of Cancer Research, London, SW3 6JB, UK.

3. Genome Stability and Human Disease Laboratory, Institute of Cancer and Genomic Sciences, University of Birmingham, Birmingham B15 2TT, UK.

4. Paediatric Immunology, Allergy and Infectious Diseases, Sheffield Children's Hospital NHS Foundation Trust, Sheffield, UK.

5. Centro Andaluz de Biología Molecular y Medicina Regenerativa CABIMER, Universidad de Sevilla-CSIC-Universidad Pablo de Olavide, Seville, Spain.

6. Division of Pediatric Hematology-Oncology, Carver College of Medicine and the University of Iowa Stead Family Children's Hospital, Iowa City, IA 52242. USA.

2. These authors contributed equally

*Correspondence addressed to: Yatin M. Vyas, E-mail: yatin-vyas@uiowa.edu. Wojciech Niedzwiedz, Email: wojciech.niedzwiedz@icr.ac.uk.

Running title: Nuclear actin counteracts replicative stress by promoting replication fork stability Key words: DNA replication, single-stranded DNA, genome stability, actin, actin nucleation 


\section{SUMMARY}

Accurate genome replication is essential for all life and a key mechanism of disease prevention, underpinned by the ability of cells to respond to replicative stress and protect stalled replication forks. All such responses rely on the formation of Replication Protein A (RPA)-ssDNA complexes, yet supra-physiological binding of RPA to ssDNA is toxic. How cells regulate RPA availability to promote fork protection and genome stability is largely unknown. Here we establish that during replication excess RPA is sequestered by monomeric actin and released upon replicative stress through transition to polymeric actin state. Impairment in actin nucleation leads to RPA sequestration, deprotection of ssDNA generated at the stressed forks and consequently, catastrophic fork collapse and hypersensitivity to replication inhibitors. In line with this, we show that increasing RPA load is sufficient to restore efficient fork protection in actin polymerization mutants. Collectively, this work identifies a simple yet robust RPA-buffering mechanism regulating its availability to bind ssDNA and protect replication forks against nucleolytic degradation. Inhibition of this pathway could be of therapeutic interest in treatment of cancers. 


\section{INTRODUCTION}

Faithful genome duplication during cell division ensures accurate transmission of genetic information to daughter cells. This process relies on the replication of the entire genetic material during S-phase by thousands of replication forks (RF) emanating from numerous origins of replication. However, replication is constantly challenged by endogenous and exogenous agents that may damage $\mathrm{RFs}^{1-3}$. To respond to replicative stress and prevent stalled forks from collapsing into DNA double-strand breaks (DSBs), a particularly toxic lesion that is mutagenic if repaired incorrectly, cells have evolved intricate genome surveillance mechanisms underpinned by the ATR and ATM kinases 2, 4,5. Mutations in genes involved in DNA replication or repair lead to human disorders with symptoms including developmental abnormalities and cancer, underscoring their importance to human health $1,6,7$.

A key step in the replication stress response (RSR) is the formation of RPA-coated single stranded DNA (ssDNA) at sites of RF damage. Replication protein A (RPA) is a heterotrimeric complex with essential roles in several aspects of DNA metabolism, with conservation from yeast to humans. During DNA replication, RPA protects ssDNA transiently formed at the stressed forks against nucleolytic degradation and serves as a platform for recruitment and regulation of a number of DNA damage response (DDR) factors, including ATR ${ }^{8,9}$. Accordingly, elegant work from the Lukas lab showed that exhaustion of the RPA pool leads to irreversible fork collapse and cell death, highlighting its protective role during replication ${ }^{10}$. However, excessive RPA accumulation at stalled forks also induces spontaneous RF breakage and this pathological outcome is supressed by the ATR kinase ${ }^{10,11}$. Indeed, RPA overexpression has been proposed to have an adverse effect on cellular and organismal fitness by facilitating tumour progression, chemotherapy-resistance, DNA-damage tolerance or compromising DNA repair fidelity ${ }^{12-14}$. Whether cells possess a mechanism to actively control/buffer free RPA (i.e., the pool of RPA that is not bound to ssDNA under normal growth conditions) is unknown and is a key question in the field. 
Polymerized actin filaments (F-actin) are major cytoskeleton components and drive multiple cellular processes from facilitating cell movement and adhesion to protein and RNA transport. Interestingly, Modulators of Actin Nucleation (MANs) are also found in the nucleus and have been shown to participate in a range of responses essential for genome maintenance. These include transcription, DNA replication and DSB repair ${ }^{15,16}$. The three major classes of MANs are the ARP2/3 complex, formins, and Spire- family proteins. ARP2/3 nucleates short branched actin filaments and is activated by the Wiskott-Aldrich Syndrome (WAS) family proteins, including WASp, N-WASp, WAVE/Scar and WASH ${ }^{17}$. Some of these factors have been implicated in R-loop resolution, relocation of stressed RFs and DSB repair by homologous recombination ${ }^{18-21}$. In contrast, formins of the DIAPH-family nucleate long unbranched actin filaments and play roles in centromere maintenance through recruitment of CenpA ${ }^{22}$ and DNA replication initiation ${ }^{23}$. Together, these findings highlight the essential role(s) of the MANs and nuclear F-actin, both branched and unbranched, in promoting genome stability. However, the molecular basis as to how actin and its nucleators regulate the RSRs remains unknown.

In this study, we find that actin nucleation controlled by WASp, N-WASp, ARP $2 / 3$ or DIAPH1 is required to promote, in trans, the generation of RPA-ssDNA complexes and protection of stalled forks from nucleolytic-degradation. Interference with actin nucleation triggers replication fork dysfunction, defective ATR activation and hypersensitivity to replication inhibitors. Remarkably, supra-physiological RPA expression suppresses degradation of nascent DNA and restores RF protection. Importantly, this is a specific response as RPA overexpression does not rescue fork instability observed in BRCA2-deficient cells. While our data do not suggest a direct role of actin polymerisation in the deposition of RPA at stressed replication forks, we propose that excess RPA is sequestered by monomeric G-actin under normal growth condition and released upon replicative stress through transition to polymeric F-actin. This allows for a rapid increase in the pool of RPA available to protect ssDNA generated at stressed forks. Thus, by constantly buffering RPA levels cells supress toxicity associated with unscheduled formation of 
RPA-ssDNA complexes under normal growth condition ${ }^{10,11}$ but importantly, retain the ability to rapidly respond to replicative stress, protect ssDNA and promote fork repair. This work also suggests that pharmacological inhibition of actin polymerization could be an effective strategy to sensitize tumours to replication inhibitors.

\section{$\underline{\text { RESULTS }}$}

\section{Actin modulators are recruited to sites of DNA replication and protect cells from replicative} stress

Recently, we employed the isolation of protein on nascent DNA (iPOND) method to detect factors involved in RF protection, to identify several proteins recruited to stalled RFs ${ }^{24-26}$. Deep mining of our data set combined with STRING analysis (http://string-db.org) identified a GO term enrichment cluster of proteins involved in actin polymerisation (Figure 1A). These included the ARP2/3 complex, WASp family proteins and members of the formin family of actin nucleators DIAPH1, 2 and 3; but not Spire family proteins.

We validated the presence of these factors at sites of ongoing replication by combining proximity-ligation assay (PLA) with EdU labelling of nascent DNA, which enables detection of the association of proteins with newly formed nascent DNA ${ }^{24,27}$. To this end, HeLa cells were labelled with EdU for $10 \mathrm{~min}$ and subsequently treated with hydroxyurea (HU) to induce replicative stress followed by PLA using antibodies specific to WASp or N-WASp to detect their enrichment at nascent DNA. We readily detected nuclear PLA signal for both proteins upon replicative stress that was significantly enriched compared to untreated as well as control samples $(\mathrm{p}<0.0001$; Figures $1 \mathrm{~B}$ and S1A). To ascertain that the observed phenotype is not restricted to EdU labelling we repeated these analyses using an antibody against PCNA - an established marker of ongoing replication. Again, we readily detected association between PCNA and the protein of 
interest (Figures S1B and C), confirming their proximity to sites of DNA replication. To further test if the proteins were part of the replisome or simply associated with chromatin, we performed a thymidine chase analysis, with thymidine addition leading to a significant decrease in PLA signal (Figure 1C). This indicates that both factors specifically associate with RFs rather than being generically recruited to chromatin. In support of this, we also observed the formation of nuclear actin filaments upon treatment with the replication inhibitor HU (Figure S1D). This observation is in agreement with previous reports of nuclear actin filament formation upon replicative stress 21,28 .

Next, we tested the effect of downregulating WASp or DIAPH1 expression or chemically inhibiting ARP2/3-mediated actin polymerisation by CK-666 treatment on cellular survival in response to treatment with replication inhibitors. Perturbation of these factors resulted in a significant decrease in survival as compared to control samples (Figure 1E), further supporting a role for actin modulators in RSR. Interestingly, this hypersensitivity is highly evolutionary conserved as yeast Saccharomyces cerevisiae mutated for the orthologue of human WASp (Las17), displayed a profound sensitivity to replication inhibitors (Han et al. accompanying manuscript). Together, this analysis reveals a putative role of MANs in mitigating replicative stress.

\section{Deficiency in actin modulators provokes global replication dysfunction}

To address the mechanism by which actin modulators promote efficient DNA replication we employed DNA fibre assay to analyse various aspects of DNA replication ${ }^{29,30}$. Strikingly, mild replicative stress induced by treatment with $\mathrm{HU}$, resulted in a highly significant reduction $(p<0.0001)$ in the average fork velocity in WASp-deficient, N-WASp-deficient, and DIAPH1deficient cells relative to WT control (Figures 2A and S2A). Importantly, this phenotype is not restricted to RNA interference as we recapitulated these observations in cells treated with an ARP2/3 inhibitor, WASp ${ }^{-/}$cells generated by CRISPR/Cas9 and a patient-derived cell-line with 
DIAPH1-mutation, compared to its re-complemented control cell line (Figures 2B and S2B). In addition, loss of WASp, N-WASp or DIAPH1 significantly impaired fork restart upon HU treatment (Figure 2C). Finally, to ascertain whether these factors cooperate in a single pathway, we tested the effect of combined ARP2/3 inhibition with WASp or DIAPH1 depletion on fork speed. Targeting either WASp or ARP2/3 alone as well as inhibiting both together resulted in a similar replication defect, consistent with the model that WASp and ARP2/3 function in the same pathway for actin polymerisation (Figure S2C). In contrast, combinatorial inhibition of ARP2/3 and DIAPH1 led to a significantly greater replication defect compared to inhibiting DIAPH1 alone (Figure 2D), indicating an additive effect between branched and unbranched actin polymerisation pathways in managing replication stress. Taken together, these data suggest that MANs of the WASp/ARP2/3 and DIAPH (formin) families are required to facilitate fork stability and global RF dynamics.

\section{Interfering with actin polymerisation elicits replication-associated DNA damage}

Unstable RFs are prone to collapse into single-ended DSBs. Thus, to determine the fate of stalled RFs when actin nucleation is impaired, we analysed the formation of 53BP1 foci, a marker of DSB induction in cells depleted for either WASp, N-WASp or DIAPH1. We noticed a significant increase in the number of 53BP1 foci in cyclin A-positive (S/G2-phase) cells after depletion of either of the three proteins as compared to their respective control cells, most likely due to extensive fork collapse (Figure 3A and S2D). A similar phenotype was observed with the use of the ARP2/3 inhibitor CK-666 and in patient derived DIAPH1 mutant cells (Figure S2D). Fork collapse is known to induce genome instability resulting in the increased formation of micronuclei. Consistently, loss of MANs resulted in increased levels of micronuclei compared to control cells even in the absence of any exogenous treatment (Figures 3B and S2E, suggesting a role for actin nucleation in preventing genome-instability from endogenous sources of DNA damage. Accordingly, cells depleted of WASp also showed increased levels of both, DAPI- 
positive chromatin bridges as well as ultra-fine anaphase bridges (UFBs), the latter of which is considered a marker of elevated replicative stress ${ }^{31}$ (Figure S2F). Collectively, these data indicate that modulators of both branched (i.e., ARP2/3-mediated) and unbranched (i.e., formin-mediated) actin polymerization promote recovery of stressed RFs and suppress replication-associated genome instability.

\section{Actin nucleation protects replication forks from uncontrolled degradation by promoting RPA localisation to ssDNA}

Unstable RFs undergo excessive nucleolytic degradation ${ }^{32-35}$ thus, we tested whether loss of MANs impacts this process. Accordingly, we employed a modified DNA fibre protocol as described recently ${ }^{26}$. Here, depletion of WASp, N-WASp or DIAPH1 resulted in a significant shortening of the CldU tracts compared to controls, indicating increased nucleolytic degradation of nascent DNA after fork arrest (Figure 4A). This phenotype was also observed in HeLa cells treated with specific siRNAs as well as $\mathrm{WASp}^{-/}$cells (Figure 4B). As pathological fork resection is attributed to the unrestrained activity of the MRE11 nuclease, we monitored fork degradation in cells treated with the MRE11 inhibitor mirin. Treatment with mirin supressed excessive fork degradation observed in cells depleted of MANs (Figure S3A). Given that MRE11 loading at stressed forks was unaffected upon WASp-depletion (Figure S3B) we concluded that the excessive fork degradation is most likely induced by fork de-protection ${ }^{36}$.

Therefore, we turned our attention to an early phase of RF repair, the loading of RPA, Rad51 or BRCA2 as this is known to be a critical step in protecting stalled forks from uncontrolled nucleolytic attack ${ }^{32,37}$. To this end, we analysed RPA foci formation upon HU treatment. Remarkably, depletion of all three actin modulators (WASp, N-WASp or DIAPH1) led to a profound defect in RPA foci upon replication stress (Figure 4B and S3C). Pharmacological inhibition of $\mathrm{ARP} / 2 / 3$ resulted in a similar defect in RPA foci formation (Figure $\mathrm{S} 3 \mathrm{C}$ ). This finding suggests impaired formation of RPA-ssDNA in the absence of the above actin modulators. 
To directly test this possibility, we analysed repair events downstream of RPA-ssDNA formation. First, we looked at loading of RAD51 at stressed forks, an event dependent on RPA-ssDNA formation ${ }^{8}$. We detected a significant defect in RAD51 association with newly replicated DNA, as measured by the PLA assay, in the absence of WASp (Figure 4C). We also observed a similar defect for BRCA2, indicating a general inability of stressed forks to initiate repair upon depletion of WASp (Figure S3D). Formation of RPA-ssDNA is also required for an ATR activation via ATRIP/RPA-dependent chromatin tethering ${ }^{9}$. Again, and in line with the defective RPA loading, ATR activity was significantly compromised as evidence by excess origin firing in WASp-, NWASp-, and DIAPH1-deficient cells (Figure 4D) as well as defective phosphorylation of CHK1 upon replication stress (Figure S3E). Altogether our data suggest two possibilities: first that MANs affect nucleolytic processing of stressed forks to generate ssDNA, but we considered it unlikely given the high rate of fork degradation; alternatively, actin modulators may have a specific role in promoting loading of RPA onto ssDNA at stressed forks. Consistent with the latter, MRE11 recruitment upon inhibition of actin polymerisation was largely unaffected (Figure S3B; in agreement with the increased fork degradation observed by DNA fibre) suggesting a specific actin-dependent process that controls RPA availability for deposition at stalled forks which is regulated by WASp- and DIAPH-family proteins.

\section{Increasing the nuclear pool of RPA ameliorates dysfunctional replication phenotypes associated with deficiency in actin nucleation}

Since RPA was recently shown to co-immunoprecipitate with actin ${ }^{38}$, we considered the possibility that targeting actin nucleators may alter the levels of "free" RPA available to bind ssDNA at stressed forks i.e. impaired F-actin generation will lead to G-actin-dependent sequestering of RPA in the nucleus. The simple prediction from this model would be that increasing the "free" nuclear RPA pool beyond the RPA:G-actin saturation stoichiometry should rescue phenotypes associated with loss of actin nucleators. To test this hypothesis, we utilised the 
SuperRPA U2OS cells generated in the Lukas lab; which express a 2 fold excess of all three RPA subunits, which does not have an adverse effect on either DNA replication or ATR activation ${ }^{10}$. First, we analysed the ability of SuperRPA cells to generate RPA-ssDNA complexes at stressed forks. Strikingly, RPA over-expression restored normal kinetics of RPA loading at damaged forks in WASp or DIAPH1 depleted cells (Figure 5A). We next analysed global RF dynamics employing the DNA fibre technique. Again, SuperRPA cells rescued the defective fork progression in WASp or DIAPH1 depleted cells (Figure 5B) with a concomitant rescue of excess fork collapse - as measured by 53BP1 foci formation (Figures 5C and S4A). Thus, remarkably, elevated levels of RPA were sufficient to rescue the phenotypes we observed associated with depletion of actin nucleators. Since, this was unexpected, we examined if elevated levels of RPA can, in general, rescue phenotypes associated with RFs instability. To test this, we analysed DNA replication in SuperRPA cells depleted for BRCA2 protein, since BRCA2-depletion confers a strong defect in fork stability/resection ${ }^{33}, 39$. However, this analysis revealed that RPA overexpression did not rescue BRCA2-associated fork instability, supporting a specific, functional interaction between RPA and actin modulators in ameliorating replicative stress (Figures S4B and C).

Our results can be explained if actin polymerisation via WASp/ARP2/3 or formindependent pathways acts early in the RSR pathway to promote the release of actin bound RPA through actin polymerisation, stimulating efficient RPA- ssDNA complex formation to protect stressed forks. However, this notion entails two predictions: first that RPA interacts directly with nuclear monomeric G-actin and secondly, disrupting actin polymerisation (i.e., a relative increase in G-actin) but not stabilizing actin filaments (i.e., increased F-actin) should impair RPA foci formation. We tested these predictions using purified proteins as well as actin mutants that either preclude (G13R) or promote (S14C) F-actin formation ${ }^{40}$. In support of our hypothesis, monomeric G-actin binds purified RPA complex directly in in vitro pull-down assays (Figure 6A). Furthermore, HU-induced RPA foci formation as well as DNA replication is impaired in cells 
expressing the polymerization-incompetent mutant G13R, whereas in the polymerizationhypercompetent S14C mutant expressing cells both, RPA foci formation and DNA replication are comparable to WT control (Figures 6B schematic, C, D and Model), thus, indicating unique cooperativity between RPA and actin polymerization dynamics to safeguard the genome.

\section{DISCUSSION}

The RPA-ssDNA-protein complex plays a key role in protecting ssDNA generated during DNA metabolism, including at stressed RFs, as well as acting as a "platform" for recruitment/signalling/regulation of a plethora of repair factors. To efficiently support these processes, cells maintain a high level of RPA in the nucleus throughout the S-phase (around 8fold excess of what is required to support unperturbed replication ${ }^{10}$ ). However, increased RPA levels can be toxic and drive genome instability and cancer progression, likely due to excessive formation of RPA-ssDNA complexes ${ }^{10,14,41-43}$. Nonetheless, the prevailing view in the field is that the RPA molecules are freely diffused throughout the nucleus and bind rapidly to available ssDNA by a passive mechanism.

Here we have uncovered an actin polymerisation dependent mechanism that buffers the availability of RPA within the nucleus and as a consequence controls, in trans, RPA:ssDNA complex formation. In line with this, we show that MAN proteins involved in ARP $2 / 3$ or formindependent actin polymerisation are specifically enriched at stressed forks and their loss inhibits RPA localisation to ssDNA resulting in fork collapse, defective activation of ATR, hypersensitivity to replication inhibitors and ultimately, increased genome instability. Furthermore, the two-actin polymerisation pathways (branched and unbranched) act in parallel as disruption of both pathways has a stronger effect on the ability of cells to protect stressed RFs. Accordingly, our work reveals an unexpected association between actin nucleation and the ability 
of cells to respond to replicative stress. While our findings with G-actin vs F-actin dominant mutants on the RPA activity (Fig. 6) would suggest that actin polymerisation is the critical mechanism impacting RPA availability. We cannot exclude the contribution of other functions of the WASp family proteins in supressing replicative stress. As such, inactivation of WASp (yeast Las17) confers a stronger phenotype compared to depletion of $\operatorname{arp} 2 / 3$ in yeast (Han et al. accompanying manuscript).

Remarkably, a supra-physiological increase in nuclear RPA by simple overexpression was sufficient to correct RPA-ssDNA complex formation and the observed replication-associated genome-destabilizing phenotypes. Furthermore, this is a specific response seen with MANdeficiency as RPA overexpression does not rescue RF defects associated with BRCA2 deficiency. Interestingly, loss of MANs has a deleterious effect on DNA replication only upon replicative stress, as rates of replication were found to be comparable under normal growth conditions upon nucleator depletion or inhibition. Thus, we propose that RPA is actively buffered in S-phase by monomeric actin and, upon replicative stress, actin polymerization drives dissociation of actinbound RPA, which in turn facilitates efficient formation of RPA-ssDNA complexes essential for fork protection and robust checkpoint activation. In support of this notion, we show that actin interacts directly with RPA and actin mutants that impede its polymerisation confer a stronger replicative phenotype. In contrast, polymerization-hypercompetent actin mutant supports efficient RPA foci formation and DNA replication (Figure 6 and Model). Recent work from the Cesare laboratory indicated a role for ATR in promoting nuclear actin filament formation ${ }^{21}$. Our findings raise an intriguing possibility that an ATR-dependent feed-forward loop links control of RPA availability with the severity of replicative stress. Such a mechanism would allow for "discreet" activation of the replication checkpoint, facilitating "local" vs "global" responses. Indeed, it has been proposed recently that the key function of checkpoint signaling is to control the global surplus of nuclear RPA, shielding RFs from catastrophic breakage ${ }^{44}$. 
Why should cells need to control RPA levels given the critical role of this complex in almost all processes related to DNA-related metabolism? There are at least two possible scenarios: firstly, the amount of nuclear RPA is several-fold higher than what is required to support unperturbed DNA replication yet overloading of RPA on ssDNA is toxic and leads to excessive fork collapse and genome instability. Thus, under non-stress conditions cells may need to efficiently control the level of free nuclear RPA to prevent pathological formation of extensive RPA-ssDNA complexes. Moreover, a robust buffering mechanism would ensure that RPA can be made rapidly available upon acute replicative stress for shielding of ssDNA as well as acting as a hub for binding of DDR factors required for repair.

Tumorigenesis is driven by elevated replication stress and interestingly, the majority of cancers overexpress actin nucleators ${ }^{45}$ suggesting that dysregulation of actin polymerisation may support oncogenic transformation. Our model proposes that a shift in the balance between G-actin to F-actin would increase the intrinsic level of free-RPA, and thus likely contribute to an enhanced ability of cancer cells to cope with elevated replicative stress/DNA damage. Indeed, high RPA levels positively correlate with tumour progression and therapeutic resistance in human cancers 46, 47. This may have important ramifications for cancer therapies employing checkpoint inhibitors and/or replicative stress-inducing agents by narrowing the effective therapeutic window (i.e. increased dynamic range of RPA response as observed in the "rescue" phenotype in our analysis of SuperRPA cells). Consequently, combinational therapy targeting both, actin dynamics to induce deficiency in RPA levels as well as DNA replication itself may prove significantly more toxic. Accordingly, recent work shows that inhibition of RPA increases the efficacy of ATR/CHK1 inhibitors ${ }^{48}$. Thus, our work highlights the possibility of targeting actin dynamics to improve existing cancer therapies.

Importantly, patient phenotypes with mutations in actin polymerisation pathways closely resemble those found in genetic disorders with defects in replication/DNA repair pathways i.e., increased cancer risk, failure of the hematopoietic system (immunodeficiency), microcephaly, 
neurodegeneration, developmental delay or premature aging, ${ }^{15,49-52}$. The severity and broad spectrum of these phenotypes likely reflect the high redundancy within these mechanisms as well as functions outside of the DDR responses. Indeed, disruption of ARP2/3 and DIAPH1-dependent actin polymerisation pathways confers a stronger repair defect than loss of a single mechanism.

The association between actin polymerisation and RSRs shown here, has at least two important implications from a clinical perspective. First, given the increased cancer predisposition in Wiskott-Aldrich Syndrome or DIAPH1-mutated patients and the fact that systemic chemotherapy is a prevalent method of cancer treatment, these patients may require a reduced intensity regimen. Secondly, a similar consideration may apply to patients undergoing conditioning regime that includes chemotherapy, prior to bone marrow transplant.

Altogether, our findings establish a fundamental mechanism by which actin controls RPA that is available for protection of ssDNA and consequently is essential for genome stability. Upon replicative stress actin polymerisation drives release of RPA bound to monomeric actin, making it available for rapid deposition and the subsequent protection of extensive stretches of ssDNA generated at stressed replication forks. This work also opens up the possibility that modulating actin polymerization may improve efficacy of cancer therapies targeting DNA replication.

\section{Acknowledgements}

We thank L. I. Toledo (University of Copenhagen) for U2OS SuperRPA cell line, M. Seidman and M. Bellini (NIH, Baltimore, USA) for scholarly input, M. Dylewska and C. Smith for technical assistance, and the Flow Cytometry \& Light Microscopy Facility at the ICR for assistance with microscopy and flow cytometry cell sorting. Work in W.N.'s laboratory is funded by ICR Intramural Grant and Cancer Research UK Programme (A24881). P.M. was supported by the BBSRC fellowship (BB/T009608/1). GSS is funded by a CR-UK Programme Grant (C17183/A23303). Y.M.V research was funded by grants from NIH, National Institute of Allergy and Infectious Diseases (NIAID) grants R01AI146380, Research Bridge Award from the Carver 
College of Medicine and the Endowment from the Mary Joy and Jerre Stead Foundation. A.A. was funded by the European Research Council (grant ERC2014 AdG669898 TARLOOP). BLW is supported through the CR-UK Clinical Academic Training Programme award (C11497/A31309).

\section{Contributions}

W.N. and Y.M.V. conceived the project. W.N., J.N. and P.M. designed and analysed the experiments with contribution from R.B.; J.N., P.M. and R.B. performed most of the experiments. J.K. and A.K. contributed with specific experiments. A.A. provided advice on project design and data analysis. C.B. and V.B. provided assistance with visualisation of actin polymers. G.S.S. provided advice on project design and carried out initial analysis and complementation of DIAPH1 cell line with assistance from B.L.W.; F.S. provided the DIAPH1 cell line. W.N. wrote the manuscript with editing contributions from J.N., R.B., P.M., Y.M.V., A.A and G.S.S., and all authors reviewed it.

The authors declare no competing interests 


\section{FIGURE LEGEND}

Figure 1

WASp, N-WASp, ARP2/3 and DIAPH1 associate with replication forks.

a) STRING analysis of iPOND MS data in untreated and HU treated HeLa S3 cells.

b) Representative images and dot plots of the number of WASp/Biotin and N-WASp/Biotin PLA foci per nucleus in HeLa cells upon HU treatment as indicated (red lines indicate mean values). Statistical significance was determined using the Mann-Whitney test.

c) Representative images and dot plots of number of WASp/Biotin and N-WASp/Biotin PLA foci per nucleus in HeLa cells treated with $1 \mathrm{mM} \mathrm{HU}$ for $1 \mathrm{~h}$, followed or not by thymidine chase as indicated (red lines indicate mean values). Statistical significance was determined using the Mann-Whitney test.

Figure 2

WASp, N-WASp, ARP2/3 and DIAPH1 are required for intact replication dynamics under condition of replicative stress.

a) Box plots of CldU/IdU tract ratios from HeLa cells treated with control siRNA or siRNA against WASp, N-WASp or DIAPH1 treated with 1mM HU during CldU labelling pulse. Whiskers indicate 5-95 percentile. Statistical significance was determined using the MannWhitney test.

b) Box plots of CldU/IdU tract ratios from WASp knock-out ND1 cells or DIAPH1 deficient patient derived cells treated with 1mM HU during CldU labelling pulse. Whiskers indicate 5-95 percentile. Statistical significance was determined using the Mann-Whitney test.

c) Box plots of CldU/IdU tract ratios of HeLa cells treated with control siRNA or siRNA against WASp, N-WASp or DIAPH1. After IdU pulse cells were exposed to 2mM HU for $2 \mathrm{~h}$ followed by release in to CldU for 60 minutes. Whiskers indicate 5-95 percentile. Statistical significance was determined using the Mann-Whitney test. 
d) Box plots of CldU/IdU tract ratios from DIAPH1 deficient patient derived cells treated or not with CK-666. During CldU labelling pulse cells were treated with 1mM HU. Whiskers indicate 5-95 percentile. Statistical significance was determined using the Mann-Whitney test.

Figure 3

Depletion of WASp, N-WASp or DIAPH1 leads to increase in replication fork collapse.

a) Representative images and dot plots of the number of 53BP1 foci per cyclin A positive HeLa cells treated with control siRNA or siRNA targeting WASp, N-WASP or DIAPH1 upon HU treatment. Statistical significance was determined using the Mann-Whitney test.

b) Representative images and quantification of HeLa cells with micronuclei treated with control siRNA or siRNA targeting WASp or N-WASp. Statistical significance was determined using $\mathrm{t}$ test.

Figure 4

Depletion of WASp, N-WASp or DIAPH1 leads to increased degradation of nascent DNA and defects in RPA recruitment to stressed replication forks.

a) Box plots of CldU/IdU tract ratios from HeLa cells treated with control siRNA or siRNA targeting WASp, N-WASP or DIAPH1 and WASP knock out ND1 cells. CldU/IdU pulse labelling was followed by treatment with $4 \mathrm{mM} \mathrm{HU}$ for $4 \mathrm{~h}$. Whiskers indicate 5-95 percentile. Statistical significance was determined using the Mann-Whitney test.

b) Representative images and dot plots of RPA signal intensity per nucleus of Hela cells treated with control siRNA or siRNA targeting WASp or N-WASP WASP, and DIAPH1 deficient patient derived cells upon HU treatment as indicated (red lines indicate mean values). Statistical significance was determined using the Mann-Whitney test. 
c) Dot plots of number of Rad51/Biotin PLA foci per nucleus in HeLa cells treated with control siRNA or siRNA against WASp (red lines indicate mean values). Statistical significance was determined using the Mann-Whitney test.

d) Quantification of the frequency of origin firing in HeLa cells treated with control siRNA or siRNA against WASp, N-WASp or DIAPH1. Statistical significance was determined using $\mathrm{t}$ test.

Figure 5

Overexpression of RPA rescues replication related defects caused by depletion of WASp or DIAPH1.

a) Representative images and dot plots of RPA signal intensity per nucleus of U2OS WT cells and U2OS cells overexpressing RPA treated with control siRNA or siRNA targeting WASp or DIAPH1 upon HU treatment as indicated (red lines and numbers above plots indicate mean values). Statistical significance was determined using the Mann-Whitney test.

b) Box plots of CldU/IdU tract ratios of U2OS WT cells and U2OS cells overexpressing RPA treated with control siRNA or siRNA targeting WASp or DIAPH1 treated with 1mM HU during CldU labelling pulse. Whiskers indicate 5-95 percentile. Statistical significance was determined using the Mann-Whitney test.

c) Representative images and dot plots of the number of 53BP1 foci per cell nucleus in U2OS WT cells and U2OS cells overexpressing RPA (red lines indicate mean values). Statistical significance was determined using the Mann-Whitney test. 
Figure 6

Monomeric actin directly binds RPA and actin polymerization is required for efficient RPA deposition at stressed replication forks.

a) RPA directly binds G-actin. WB of RPA in vitro pull down with G-Actin beads.

b) Schematic depicting putative role of actin polymerization in increasing the available pool of RPA upon replicative stress (top panel); Actin G13R mutant that renders actin incompetent of polymerization prevents the release of actin bound RPA, impeding RPA deposition at stressed replication forks (left bottom panel); Actin S14C mutation which stabilizes actin filaments counteracts RPA sequestration by monomeric actin (right bottom panel)C - RPA in HeLa cells overexpressing YFP Actin and its mutants.

c) Representative images and dot plot of RPA signal intensity in HeLa cells expressing YFPactin WT, G13R or S14C mutant upon HU treatment as indicated.

d) Box plots of CldU/IdU tract ratios of HeLa cells expressing YFP-actin WT, G13R or S14C mutant proteins treated with $1 \mathrm{mM}$ HU during CldU labelling pulse. Whiskers indicate 595 percentile. Statistical significance was determined using the Mann-Whitney test.

e) Proposed model for the role of actin polymerization in the replicative stress response. Replicative stress leads to fork stalling and generation of ssDNA, nuclear actin nucleation leads to release of actin bound RPA which in turn makes it available for deposition on ssDNA generated at stressed replication forks. This facilitates efficient fork restart and promotes genome stability. Inhibition of actin polymerization impairs RPA release and the subsequent loading on ssDNA leading to extensive nascent strand degradation and ultimately adversely impacting genome stability. 


\section{METHODS}

\section{Cell lines}

HeLa cells were a generous gift from Dr F. Esashi. U2OS cells and U2OS over expressing RPA (Super RPA) cells were a kind gift of Dr L. Toledo. These cell lines were cultured in Dulbecco's modified Eagle's medium (DMEM) supplemented with $10 \%$ fetal bovine serum (FBS) and standard antibiotics. Primary dermal fibroblasts were maintained in Dulbecco's modified Eagle's medium (DMEM; Life Technologies) supplemented with $20 \%$ fetal calf serum (FCS), 5\% Lglutamine, and 5\% penicillin-streptomycin (Invitrogen) antibiotics. Primary fibroblasts were immortalized with 293FT (Invitrogen)-derived supernatant containing a human telomerase reverse transcriptase (TERT) lentivirus that was generated with the plasmids pLV-hTERT-IREShygro (gift from Tobias Meyer; Addgene $\# 85140)^{53}$, psPax2 (gift from Didier Trono; Addgene \#12260), and pMD2.G (gift from Didier Trono; Addgene \#12259). Selection was performed with hygromycin (Invitrogen) at $70 \mathrm{mg} / \mathrm{mL}$. Fibroblast complementation was carried out using a lentiviral vector (pLVX-IRES-Neo; TakaraBio) that encoded 3xHA-tagged DIAPH1 in combination with the lentiviral packaging plasmids described above. Selection was performed with geneticin (Invitrogen) at $400 \mathrm{mg} / \mathrm{mL}$. Expression of HA-tagged DIAPH1 was validated by Western blotting. All cell lines were routinely tested for mycoplasma.

\section{Plasmids}

Plasmids encoding YFP NLS Beta-Actin, YFP NLS Beta-Actin S14C and YFP NLS Beta-Actin G13R were a gift from Primal de Lanerolle (Addgene plasmid \# 60613 ; http://n2t.net/addgene:60613 ; RRID:Addgene_60613; Addgene plasmid \# 60614 ; http://n2t.net/addgene:60614 ; RRID:Addgene_60614; Addgene plasmid \# 60615 ; http://n2t.net/addgene:60615 ; RRID:Addgene_60615 respectively) ${ }^{54}$. Nuclear ActinChromobody ${ }^{\circledR} \quad($ TagGFP2) plasmid construct (acg-n, Chromotek) was purchased from Chromotek. p11d-tRPA(123) plasmid was a kind gift from David Cortez. 


\section{Immunoblotting}

Cell lysis was carried out in urea buffer $(9 \mathrm{M}$ urea, $50 \mathrm{mM}$ Tris HCL, $\mathrm{pH} 7.5,150 \mathrm{mM} \beta$ mercaptoethanol) followed by sonication using a soniprep 150 (MSE) probe sonicator. Cell lysates were prepared in SDS loading buffer (2\% SDS, 10\% (v/v) glycerol, 2\% 2-Mercaptoethanol and $62.5 \mathrm{mM}$ Tris- $\mathrm{HCl}, \mathrm{pH}$ 6.8) followed by boiling at $95^{\circ} \mathrm{C}$ for $10 \mathrm{~min} .20$ Protein concentrations were determined by spectrophotometry using a Denovix ds-11 fx + device (Denovix). Samples were resolved by SDS-PAGE and transferred to PVDF or nitrocellulose followed by blocking in $5 \%$ low fat milk in $1 \mathrm{x}$ TBS/ $0.1 \%$ Tween-20 for $1 \mathrm{~h}$ at room temperature. Membranes were washed $3 \times 5 \mathrm{~min}$ in $1 \mathrm{x} \mathrm{TBS} / 0.1 \%$ Tween-20 and incubated overnight at $4{ }^{\circ} \mathrm{C}$ in the indicated primary antibodies in 5\% low fat milk in $1 \mathrm{x}$ TBS/ $0.1 \%$ Tween-20. Membranes were subsequently washed $3 \times 5 \mathrm{~min}$ in $1 \mathrm{x}$ TBS/ $0.1 \%$ Tween-20 and incubated in 5\% low fat milk in $1 \times$ TBS/ $0.1 \%$ Tween20 containing secondary antibodies for $1 \mathrm{~h}$ at room temperature. Membranes were subsequently washed 3 x 5 min in 1x TBS/ 0.1\% Tween-20 and developed using Immobilon Western HRP Substrate (Millipore, WBKLS0S00) and exposed to X-ray film. Primary antibodies used were: $\alpha$ Tubulin (Sigma, B-5-1-2; T5168, 1:100,000), MCM2 (Abcam, ab4461, 1:10,000), N-WASp (Abcam ab187527, 1:1000), DIAPH1 (Bethyl, A300-077A-T, 1:1000; Santa Cruz , sc-373807, 1:500), CHK1 - phospho S345 (Cell Signalling, 133D3; 2348, 1:1000), CHK1 (Santa Cruz, sc8408, 1:1000. Secondary antibodies used were anti-mouse IgG-HRP (Dako, P0447, 1:2000) and anti-rabbit IgG-HRP (Dako, P0448, 1:5000).

\section{Cell Survival Assay}

Alamar Blue survival assays were performed in accordance with the manufacturer's recommendations (Bio Rad). Briefly, 500 cells per well in 96-well plates were plated and untreated or treated with indicated doses of hydroxyurea, cis-platin or Mitomycin C and incubated 
for 7 days. Alamar blue reagent was added to each well and fluorometric measurements taken after $4 \mathrm{~h}$ incubation at $37^{\circ} \mathrm{C}$.

\section{RNAi treatment}

siRNAs used in this study were as follows: siWASp - GAGUGGCUGAGUUACUUGC; siNWASp - ON-TARGETplus WASL siRNA (L-006444-00-0005, Dharmacon); siDIAPH1- ONTARGETplus DIAPH1 siRNA (L-010347-00-0005, Dharmacon); siRNA targeting luciferase CGTACGCGGAATACTTCGA ${ }^{55}$ was used as control siRNA. Oligonucleotides were transfected using HiPerfect reagent (Qiagen), according to the manufacturer's protocol.

\section{Immunofluorescence microscopy}

Cells were grown and treated on circular $13 \mathrm{~mm}$ diameter coverslips. Visualization of 53BP1 foci in all cells and in Cyclin A-positive cells, respectively, cells were fixed with 4\% paraformaldehyde in PBS for $10 \mathrm{~min}$ at room temperature, washed twice in PBS and permeabilised with $0.2 \%$ Triton X-100 in PBS for 10 min at room temperature. Coverslips were washed $3 \mathrm{x}$ in PBS cells and were blocked in 10\% FBS in PBS for $1 \mathrm{~h}$ at room temperature before incubation with primary antibodies in $0.1 \%$ FBS in PBS overnight at $4{ }^{\circ} \mathrm{C}$. Coverslips were then washed $4 \times 5$ min in PBS followed by incubation with secondary antibodies for $1 \mathrm{~h}$ at room temperature. Slides were then washed $4 \times 5 \mathrm{~min}$ in PBS and subsequently mounted with Vectashield mounting medium (Vector Laboratories) with DAPI. Micronuclei were quantified by assessing DAPI stained nuclei. For visualization of RPA2 foci, cells were pre-extracted on ice for 2 min in CSK buffer (10 mM PIPES pH6.8, $300 \mathrm{mM}$ sucrose, $100 \mathrm{mM} \mathrm{NaCl}, 1.5 \mathrm{mM} \mathrm{MgCl} 2,0.5 \%$ TritonX-100), washed with PBS and fixed with $4 \%$ paraformaldehyde in PBS for 10 min at room temperature. Coverslips were then blocked and processed as above. Primary antibodies employed for immunofluorescence were as follows: 53BP1 (MAB3802, Millipore, 1:1000), Cyclin A (ab19150, Abcam, 1:500), RPA2 (NA-18, Calbiochem, 1:200). Secondary antibodies for immunofluorescence were as 
follows: Alexa Fluor 488 anti-rabbit (A21206, Invitrogen, 1:200) and Alexa Fluor 555 anti-mouse (A31570, Invitrogen, 1:400). Images were acquired using a Leica SP8 confocal microscope with a 63x oil objective. Quantification was carried out using FIJI (ImageJ) software and CellProfiler (Broad Institute).

Nuclear actin chromobody stable cell line generation and nuclear actin aggregate quantification.

Cell lines stably expressing nuclear Actin-Chromobody® (TagGFP2) were generated by transfection of U2OS cells with the nuclear Actin-Chromobody® plasmid (TagGFP2) plasmid construct (acg-n, Chromotek) followed by selection of cells for 14 days in media containing 500 $\mu \mathrm{g}$ ml-1 G418 (Life Technologies), replacing selective media every 48-72 hours. GFP positive U2OS cells were subsequently sorted using the FACS Aria III flow cytometer (BD Biosciences). For assessment of nuclear actin chromobody, cells were grown and treated on circular $13 \mathrm{~mm}$ diameter, $1.5 \mathrm{~mm}$ thickness coverslips. Cells were washed twice in PBS and fixed with 4\% paraformaldehyde in PBS for $10 \mathrm{~min}$ at room temperature, washed $3 \times 5 \mathrm{~min}$ in $\mathrm{PBS}$ and subsequently mounted with Vectashield mounting medium (Vector Laboratories) with DAPI. A Z-series of $0.3 \mu \mathrm{M}$ intervals throughout the nuclei of cells was acquired using a Leica SP8 confocal microscope using a 63x oil objective. Quantification of nuclear actin positive cells was carried out by assessing the presence of filamentous aggregates.

\section{EdU labeling of nascent DNA and Proximity Ligation Assay}

Analysis of the association of proteins to nascent DNA by EdU labeling and the Proximity Ligation Assay was carried out as previously described ${ }^{26,27}$. Briefly, cells grown on coverslips were labeled with $10 \mathrm{mM}$ EdU for $10 \mathrm{~min}$ followed by treatment with $1 \mathrm{mM}$ or $4 \mathrm{mM}$ hydroxyurea at various timepoints as indicated. Fixation was carried out with $3 \%$ formaldehyde, $2 \%$ sucrose in PBS for $10 \mathrm{~min}$ at room temperature. Slides were then washed twice with PBS and incubated with 
blocking solution (3\% BSA in PBS) for 30 min. Following this, slides were washed 2x in PBS before EdU-Biotin Azide conjugation by click chemistry using the Click-iT reaction (Click-iT assay kit (Thermo Fisher, according to the manufacturer's instructions). Coverslips were washed $2 \mathrm{x}$ with PBS before primary antibody incubation overnight at $4 \mathrm{C}$ in $1 \% \mathrm{BSA} / 0.1 \%$ saponin in PBS. Following primary antibody incubation coverslips were washed $2 x$ in PBS and then the proximity ligation assay was carried out (Duolink In Situ Red Starter kit (Sigma Aldrich) according to the manufacturer's instructions). Coverslips were mounted using Vectashield containing DAPI. Antibodies employed for the PLA assay were as follows: Biotin (Bethyl Laboratories, A150-109A, 1:3000), (Biotin (Jackson Immunoresearch, 200-002-211, 1:1000), WASp (Santa Cruz, sc-5300, 1:500), N-WASp (Abcam, ab187527, 1:500), PCNA (Santa Cruz, sc-56, 1:500), and PCNA (Abcam, ab18197, 1:500). Images were acquired using a 3i Advance spinning disc microscope system using a $63 \mathrm{x}$ objective. Image analysis was carried out with FIJI (ImageJ) software.

\section{DNA fibre analysis}

DNA fibre assay was performed as described previously with some modifications ${ }^{26,56,57}$. In brief, exponentially growing cells were first incubated with $25 \mu \mathrm{M}$ iododeoxyuridine (IdU) and then with $125 \mu \mathrm{M}$ chlorodeoxyuridine (CldU) for the indicated times. Fibre spreads were prepared from $0.5 \times 10^{6}$ cells $/ \mathrm{ml}$. Slides were stained as described previously ${ }^{56,57}$. Images were acquired with Leica SP8 or Carl Zeiss LSM 710 Meta confocal microscope using a 63x oil objective. Analysis was performed using the ImageJ software package (National Institutes of Health). A minimum of 100 fibres from three, unless stated otherwise, independent experiments was scored. Mann-Whitney test was used to determine statistical significance.

\section{QPCR}

RNA extraction and cDNA synthesis were performed using the RNeasy mini kit (Qiagen) and 
High Capacity cDNA Reverse Transcription Kit (Applied Biosystems). QPCR analysis was performed with QuantStudio ${ }^{\mathrm{TM}} 6$ Flex Real-Time PCR System (Applied Biosystems) using SYBR Green PCR Master Mix (Life Technologies) and the following primers GTCCTACTTCATCCGCCTTTAC and TCGTCTGCAAAGTTCAGCC for WASP and GGCATGGACTGTGGTCATGAG and TGCACCACCAACTGCTTAGC for GAPDH.

\section{RPA complex expression, purification and in vitro pull down}

RPA complex was expressed from plasmid (a kind gift from David Cortez (Vanderbilt University, Nashville, Tennessee, US) ${ }^{58,59}$ caring genes encoding 6xHis-tagged RPA1 (70 kDa), tagless RPA2 (32 kDa), and 6xHis-tagged RPA3 (14 kDa) in BL21 (D3) E. coli cells for $2 \mathrm{~h}$ at $37^{\circ} \mathrm{C}$ in LB medium with $100 \mu \mathrm{M} / \mathrm{ml}$ ampicillin after induction with $1 \mathrm{mM}$ IPTG. Cells were harvested and lysed in buffer containing $50 \mathrm{mM}$ Tris-HCl, $\mathrm{pH} 8.0,400 \mathrm{mM} \mathrm{NaCl}, 1 \mathrm{mM}$ PMSF, $10 \mu \mathrm{M}$ $\mathrm{ZnCl}_{2}, 5 \%$ glycerol and protease inhibitor cocktail (Sigma, \#S8830). Supernatant was applied to a HisTrap HP $5 \mathrm{ml}$ column (DE Healthcare) calibrated in 5 volumes of calibration buffer containing $20 \mathrm{mM}$ Tris-HCl, $\mathrm{pH}$ 8.0, $500 \mathrm{mM} \mathrm{NaCl}, 1 \mathrm{mM}$ PMSF, $10 \mu \mathrm{M} \mathrm{ZnCl}_{2}$ and 5\% glycerol. Next, the column was washed in 10 column volumes with washing buffer: $20 \mathrm{mM}$ Tris- $\mathrm{HCl}$, $\mathrm{pH}$ 7.6, $500 \mathrm{mM} \mathrm{NaCl}, 20 \mathrm{mM}$ imidazole, $10 \mu \mathrm{M} \mathrm{ZnCl}_{2}$ and $5 \%$ glycerol. Then, the proteins were eluted from column with the linear imidazole gradient in final concertation as follow, $20 \mathrm{mM}$ Tris$\mathrm{HCl}, \mathrm{pH} 7.5,100 \mathrm{mM} \mathrm{NaCl}, 300 \mathrm{mM}$ imidazole, $10 \mu \mathrm{M} \mathrm{ZnCl}_{2}$ and 5\% glycerol. The RPA complex of three proteins was bound to the column and was eluted from it by high imidazole concentration (about $250 \mathrm{mM}$ ). The eluate was diluted to bring the $\mathrm{NaCl}$ concentration down to $50 \mathrm{mM}$. It was then applied to a HiTrap Heparin HP $5 \mathrm{ml}$ column (GE Healthcare) in $20 \mathrm{mM}$ Tris$\mathrm{HCl}, \mathrm{pH} 7.5,50 \mathrm{mM} \mathrm{NaCl}, 1 \mathrm{mM}$ PMSF, $10 \mu \mathrm{M} \mathrm{ZnCl}_{2}$ and $5 \%$ glycerol, and eluted with 10 volumes of $50-1000 \mathrm{mM} \mathrm{NaCl}$ linear gradient. The fractions containing all three proteins were joined together and purified RPA complex was rebuffered into storage buffer (50 mM Tris-HCl, $\mathrm{pH}$ 8.0, $100 \mathrm{mM} \mathrm{NaCl}, 1 \mathrm{mM}$ DTT, 5\% glycerol), aliquoted and frozen in $-80^{\circ} \mathrm{C}$. The complex composition was confirmed by western blot using antibodies recognizing His-tag peptide and 
RPA2 protein and by binding RPA complex to ssDNA and lack of biding it to dsDNA. Interaction of RPA complex with monomeric actin was analysed using G-Actin Sepharose beads (Hypermol). Reaction was performed in a buffer containing (10mM Tris- $\mathrm{HCl} \mathrm{pH} 7.5,150 \mathrm{mM} \mathrm{NaCl}, 1 \mathrm{mM}$ DTT, 5\% glycerol, $0.1 \%$ NP-40). Complexes were extensively washed with IP buffer before elution in 2X SDS sample buffer and subsequently boiled for $3 \mathrm{~min}$ followed by centrifugation. The resultant supernatant fraction was retained as the eluate.

\section{STRING analysis}

Gene accession codes from IPOND analysis were input into the multiple protein search function of STRING

\section{(https://string-db.org/cgi/input?sessionId=b5U7THWxoyjd\&input_page_active_form=}

multiple_identifiers) with the detected organisms selected as Homo sapiens. The network was then exported from STRING and imported into Cytoscape (v3.8.2). Functional network analysis was then conducted using the STRING plug-in. The generated network was filtered by GOprocesses. GO processes associated with actin related processes were selected and copied to a new network. The subsequently generated network was saved and the file was uploaded to STRING (https://string-db.org/cgi/input?sessionId=b5U7THWxoyjd\&input_page_show_search=on).

Active interaction sources settings were adjusted to display links associated with only experiments and databases. The minimum required interaction score was adjusted to high confidence $(0.700)$ and the network was updated. GO terms GO:0034314 Arp2/3 complex-mediated actin nucleation and GO:0008154 Actin polymerization or depolymerization were selected in the analysis tab functional enrichment biological process list.

\section{Statistics and reproducibility}

Statistical analyses were done using GraphPad Prism 9 (GraphPad Software Inc.) or Microsoft 
Excell. Unpaired Students' t-test or Mann Whitney test were used to determine statistical significance as indicated in the Figure Legends.

\section{References:}

1. Jackson, S.P. \& Bartek, J. The DNA-damage response in human biology and disease. Nature 461, 1071-1078 (2009).

2. Zeman, M.K. \& Cimprich, K.A. Causes and consequences of replication stress. Nature cell biology 16, 2-9 (2014).

3. Kotsantis, P., Petermann, E. \& Boulton, S.J. Mechanisms of Oncogene-Induced Replication Stress: Jigsaw Falling into Place. Cancer Discov 8, 537-555 (2018).

4. Cimprich, K.A. \& Cortez, D. ATR: an essential regulator of genome integrity. Nature reviews. Molecular cell biology 9, 616-627 (2008).

5. Neelsen, K.J. \& Lopes, M. Replication fork reversal in eukaryotes: from dead end to dynamic response. Nature reviews. Molecular cell biology 16, 207-220 (2015).

6. Ciccia, A. \& Elledge, S.J. The DNA damage response: making it safe to play with knives. Molecular cell 40, 179-204 (2010).

7. Kass, E.M., Moynahan, M.E. \& Jasin, M. When Genome Maintenance Goes Badly Awry. Molecular cell 62, 777-787 (2016).

8. Bhat, K.P. \& Cortez, D. RPA and RAD51: fork reversal, fork protection, and genome stability. Nature structural \& molecular biology 25, 446-453 (2018).

9. Saldivar, J.C., Cortez, D. \& Cimprich, K.A. The essential kinase ATR: ensuring faithful duplication of a challenging genome. Nature reviews. Molecular cell biology 18, 622-636 (2017).

10. Toledo, L.I. et al. ATR prohibits replication catastrophe by preventing global exhaustion of RPA. Cell 155, 1088-1103 (2013).

11. Caldwell, C.C. \& Spies, M. Dynamic elements of replication protein $A$ at the crossroads of DNA replication, recombination, and repair. Crit Rev Biochem Mol Biol 55, 482-507 (2020).

12. Marechal, A. \& Zou, L. RPA-coated single-stranded DNA as a platform for posttranslational modifications in the DNA damage response. Cell Res 25, 9-23 (2015).

13. VanderVere-Carozza, P.S. et al. Chemical exhaustion of RPA in cancer treatment. bioRxiv, 2020.2011.2030.404640 (2020).

14. Deng, S.K., Gibb, B., de Almeida, M.J., Greene, E.C. \& Symington, L.S. RPA antagonizes microhomology-mediated repair of DNA double-strand breaks. Nature structural \& molecular biology 21, 405-412 (2014).

15. Hurst, V., Shimada, K. \& Gasser, S.M. Nuclear Actin and Actin-Binding Proteins in DNA Repair. Trends Cell Biol 29, 462-476 (2019).

16. Caridi, C.P., Plessner, M., Grosse, R. \& Chiolo, I. Nuclear actin filaments in DNA repair dynamics. Nature cell biology 21, 1068-1077 (2019).

17. Pollard, T.D. Actin and Actin-Binding Proteins. Cold Spring Harb Perspect Bio/ 8 (2016).

18. Schrank, B.R. et al. Nuclear ARP2/3 drives DNA break clustering for homology-directed repair. Nature 559, 61-66 (2018).

19. Sarkar, K. et al. R-loops cause genomic instability in T helper lymphocytes from patients with Wiskott-Aldrich syndrome. J Allergy Clin Immunol 142, 219-234 (2018). 
20. Caridi, C.P. et al. Nuclear F-actin and myosins drive relocalization of heterochromatic breaks. Nature 559, 54-60 (2018).

21. Lamm, N. et al. Nuclear F-actin counteracts nuclear deformation and promotes fork repair during replication stress. Nature cell biology 22, 1460-1470 (2020).

22. Liu, C., Zhu, R. \& Mao, Y. Nuclear Actin Polymerized by mDia2 Confines Centromere Movement during CENP-A Loading. iScience 9, 314-327 (2018).

23. Parisis, N. et al. Initiation of DNA replication requires actin dynamics and formin activity. EMBO J 36, 3212-3231 (2017).

24. Higgs, M.R. et al. BOD1L Is Required to Suppress Deleterious Resection of Stressed Replication Forks. Molecular cell 59, 462-477 (2015).

25. Coquel, F. et al. SAMHD1 acts at stalled replication forks to prevent interferon induction. Nature 557, 57-61 (2018).

26. Nieminuszczy, J. et al. EXD2 Protects Stressed Replication Forks and Is Required for Cell Viability in the Absence of BRCA1/2. Mol Cell 75, 605-619 e606 (2019).

27. Taglialatela, A. et al. Restoration of Replication Fork Stability in BRCA1- and BRCA2Deficient Cells by Inactivation of SNF2-Family Fork Remodelers. Mol Cell 68, 414-430 e418 (2017).

28. Johnson, M.A., Sharma, M., Mok, M.T. \& Henderson, B.R. Stimulation of in vivo nuclear transport dynamics of actin and its co-factors IQGAP1 and Rac1 in response to DNA replication stress. Biochim Biophys Acta 1833, 2334-2347 (2013).

29. Schwab, R.A. et al. The Fanconi Anemia Pathway Maintains Genome Stability by Coordinating Replication and Transcription. Molecular cell 60, 351-361 (2015).

30. Blackford, A.N. et al. TopBP1 Interacts with BLM to Maintain Genome Stability but Is Dispensable for Preventing BLM Degradation. Molecular cell 57, 1133-1141 (2015).

31. Liu, Y., Nielsen, C.F., Yao, Q. \& Hickson, I.D. The origins and processing of ultra fine anaphase DNA bridges. Curr Opin Genet Dev 26, 1-5 (2014).

32. Hashimoto, Y., Ray Chaudhuri, A., Lopes, M. \& Costanzo, V. Rad51 protects nascent DNA from Mre11-dependent degradation and promotes continuous DNA synthesis. Nat Struct Mol Biol 17, 1305-1311 (2010).

33. Schlacher, K. et al. Double-strand break repair-independent role for BRCA2 in blocking stalled replication fork degradation by MRE11. Cell 145, 529-542 (2011).

34. Karanja, K.K., Lee, E.H., Hendrickson, E.A. \& Campbell, J.L. Preventing over-resection by DNA2 helicase/nuclease suppresses repair defects in Fanconi anemia cells. Cell cycle 13, 1540-1550 (2014).

35. Lachaud, C. et al. Ubiquitinated Fancd2 recruits Fan1 to stalled replication forks to prevent genome instability. Science 351, 846-849 (2016).

36. Berti, M., Cortez, D. \& Lopes, M. The plasticity of DNA replication forks in response to clinically relevant genotoxic stress. Nature reviews. Molecular cell biology 21, 633-651 (2020).

37. Kolinjivadi, A.M. et al. Moonlighting at replication forks - a new life for homologous recombination proteins BRCA1, BRCA2 and RAD51. FEBS Lett 591, 1083-1100 (2017).

38. Pfitzer, L. et al. Targeting actin inhibits repair of doxorubicin-induced DNA damage: a novel therapeutic approach for combination therapy. Cell Death Dis 10, 302 (2019).

39. Chaudhuri, A.R. et al. Erratum: Replication fork stability confers chemoresistance in BRCA-deficient cells. Nature 539, 456 (2016).

40. Posern, G., Sotiropoulos, A. \& Treisman, R. Mutant actins demonstrate a role for unpolymerized actin in control of transcription by serum response factor. Mol Biol Cell 13, 4167-4178 (2002). 
41. Brosh, R.M., Jr. DNA helicases involved in DNA repair and their roles in cancer. Nat Rev Cancer 13, 542-558 (2013).

42. Velegzhaninov, I.O. et al. Radioresistance, DNA Damage and DNA Repair in Cells With Moderate Overexpression of RPA1. Front Genet 11, 855 (2020).

43. Zhang, Y. \& Yu, C. Distinct expression and prognostic values of the replication protein A family in gastric cancer. Oncol Lett 19, 1831-1841 (2020).

44. Toledo, L., Neelsen, K.J. \& Lukas, J. Replication Catastrophe: When a Checkpoint Fails because of Exhaustion. Molecular cell 66, 735-749 (2017).

45. Yang, X. \& Lin, Y. Functions of nuclear actin-binding proteins in human cancer. Oncol Lett 15, 2743-2748 (2018).

46. Dahai, Y., Sanyuan, S., Hong, L., Di, Z. \& Chong, Z. A relationship between replication protein $A$ and occurrence and prognosis of esophageal carcinoma. Cell Biochem Biophys 67, 175-180 (2013).

47. Givalos, N. et al. Replication protein $A$ is an independent prognostic indicator with potential therapeutic implications in colon cancer. Mod Pathol 20, 159-166 (2007).

48. Glanzer, J.G. et al. RPA inhibition increases replication stress and suppresses tumor growth. Cancer Res 74, 5165-5172 (2014).

49. Simon, D.N., Zastrow, M.S. \& Wilson, K.L. Direct actin binding to A- and B-type lamin tails and actin filament bundling by the lamin A tail. Nucleus 1, 264-272 (2010).

50. Ercan-Sencicek, A.G. et al. Homozygous loss of DIAPH1 is a novel cause of microcephaly in humans. Eur J Hum Genet 23, 165-172 (2015).

51. Al-Maawali, A. et al. Novel loss-of-function variants in DIAPH1 associated with syndromic microcephaly, blindness, and early onset seizures. Am J Med Genet A 170A, 435-440 (2016).

52. Moulding, D.A., Record, J., Malinova, D. \& Thrasher, A.J. Actin cytoskeletal defects in immunodeficiency. Immunol Rev 256, 282-299 (2013).

53. Hayer, A. et al. Engulfed cadherin fingers are polarized junctional structures between collectively migrating endothelial cells. Nat Cell Biol 18, 1311-1323 (2016).

54. Chang, L. et al. Herpesviral replication compartments move and coalesce at nuclear speckles to enhance export of viral late mRNA. Proc Natl Acad Sci U S A 108, E136-144 (2011).

55. Tuschl, T. Cotransfection of Luciferase Reporter Plasmids with siRNA Duplexes. CSH protocols 2006 (2006).

56. Nieminuszczy, J., Schwab, R.A. \& Niedzwiedz, W. The DNA fibre technique - tracking helicases at work. Methods 108, 92-98 (2016).

57. Schwab, R.A. \& Niedzwiedz, W. Visualization of DNA replication in the vertebrate model system DT40 using the DNA fiber technique. J Vis Exp, e3255 (2011).

58. Bhat, K.P., Betous, R. \& Cortez, D. High-affinity DNA-binding domains of replication protein A (RPA) direct SMARCAL1-dependent replication fork remodeling. J Biol Chem 290, 4110-4117 (2015).

59. Henricksen, L.A., Umbricht, C.B. \& Wold, M.S. Recombinant replication protein A: expression, complex formation, and functional characterization. J Biol Chem 269, 1112111132 (1994). 


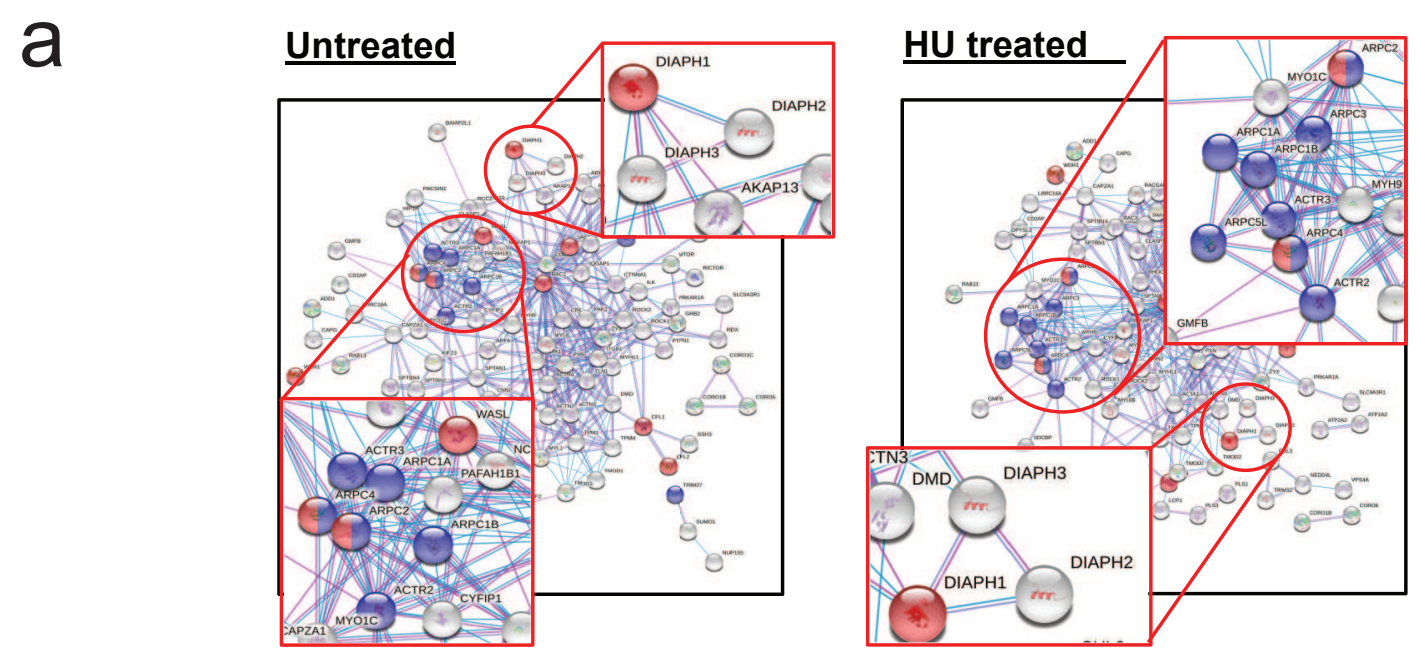

b

\begin{tabular}{|c|l|l|}
\hline GO-term & Biological Process (Gene Ontology) & $\begin{array}{l}\text { Node } \\
\text { Colour }\end{array}$ \\
\hline GO:0034314 & Arp2/3 complex mediated actin nucleation & \\
\hline GO:0008154 & Actin polymerization or depolymerization & \\
\hline
\end{tabular}

PLA: anti-WASp / anti-Biotin

PLA: anti-N-WASp / anti-Biotin

HU:
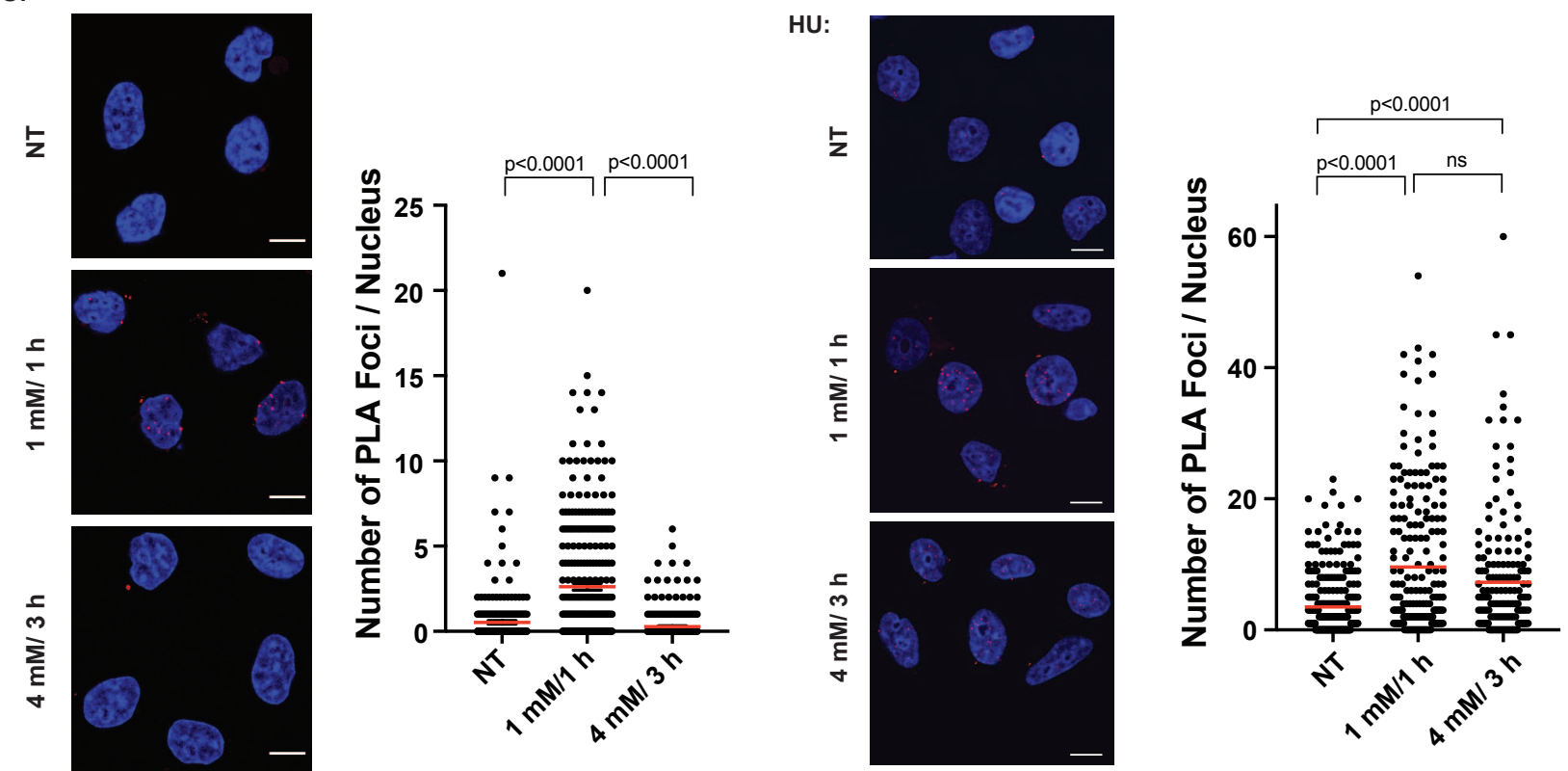

PLA DAPI

C

HU:
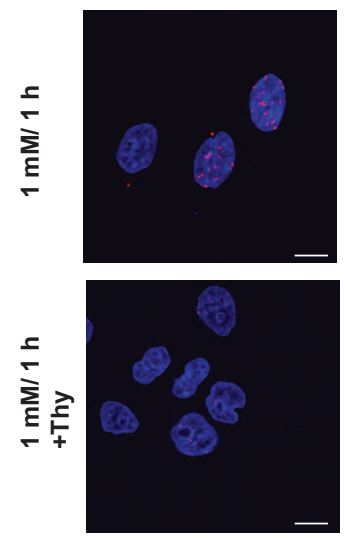

PLA DAPI
PLA: anti-WASp / anti-Biotin

Fig.1

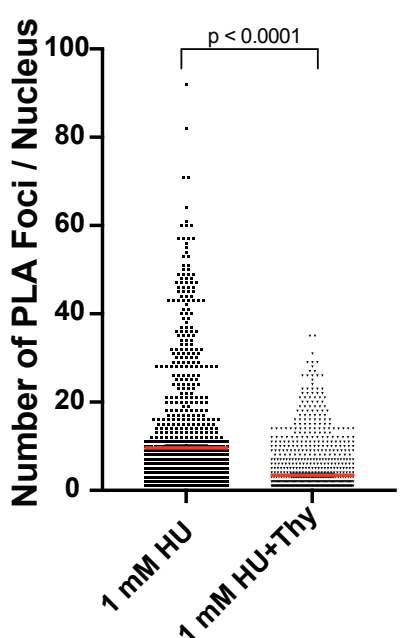

HU:

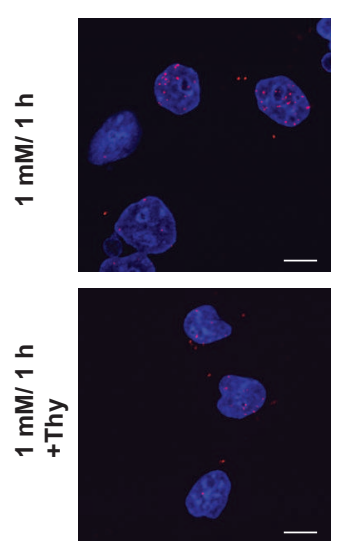



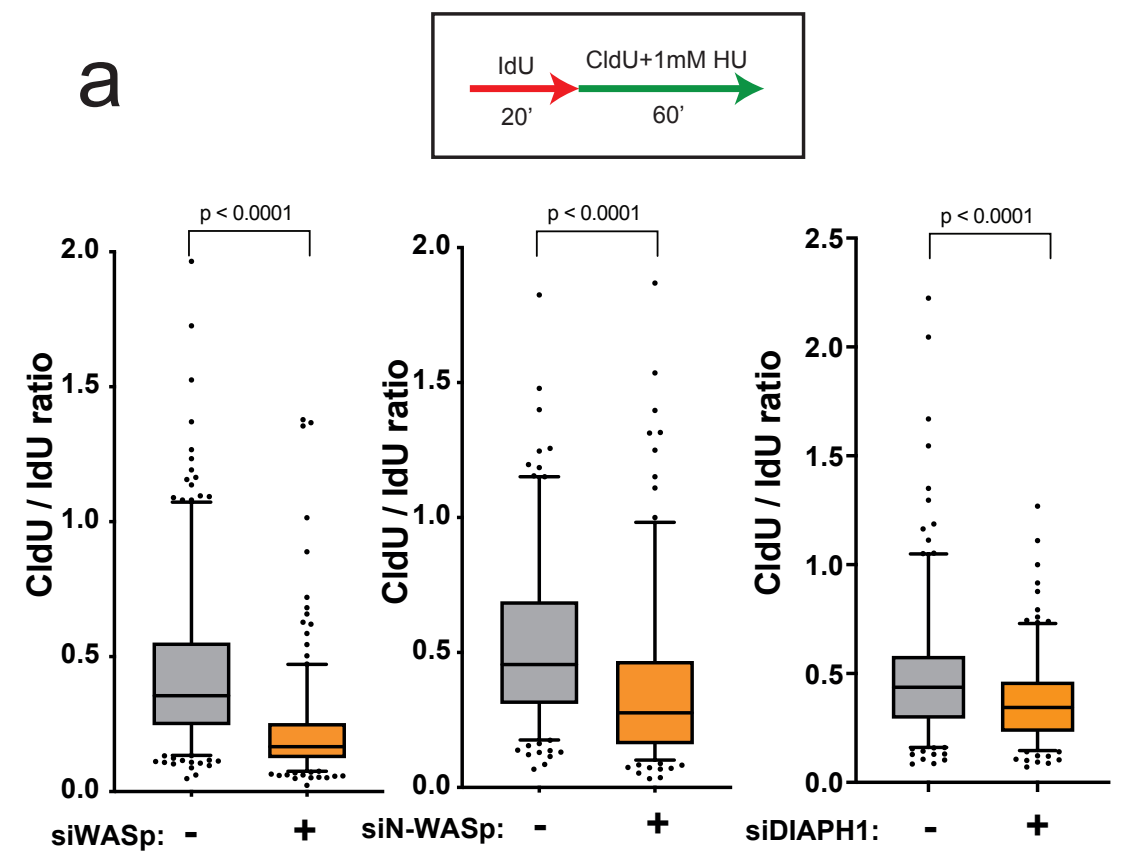

C
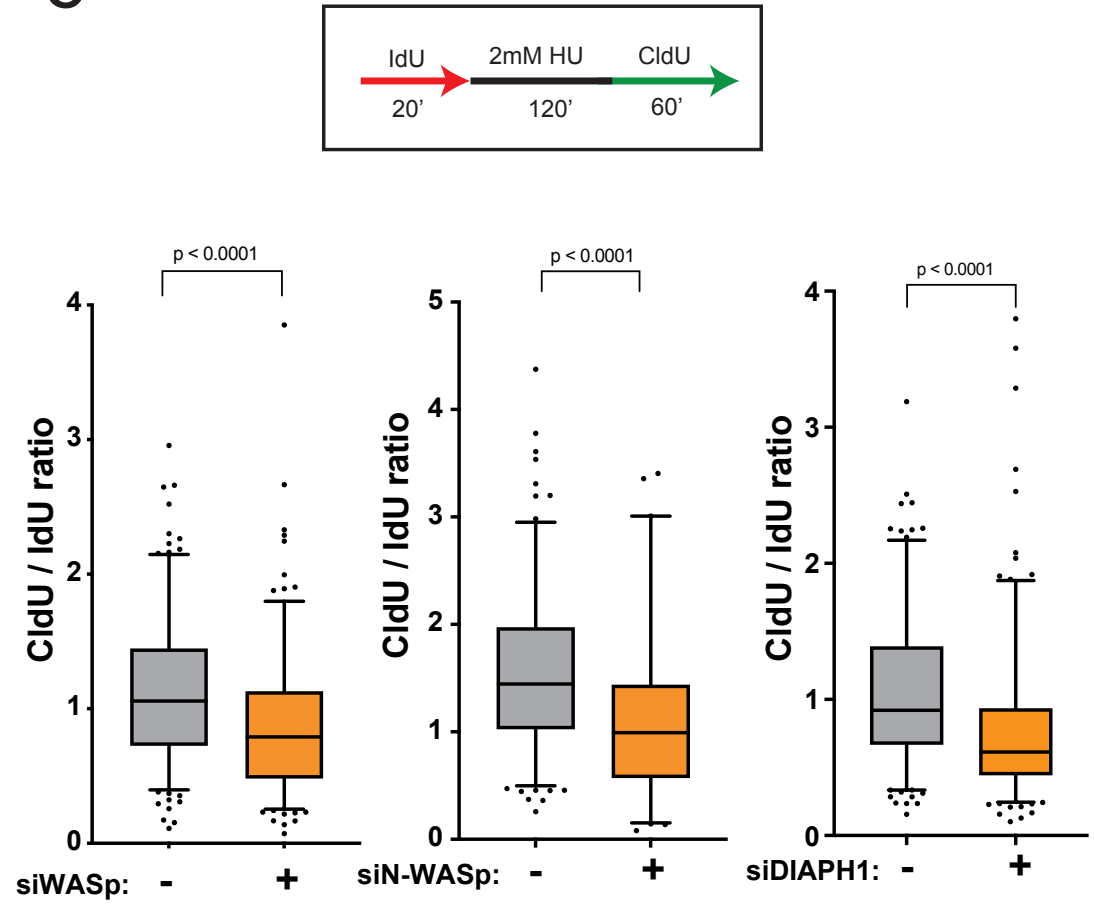
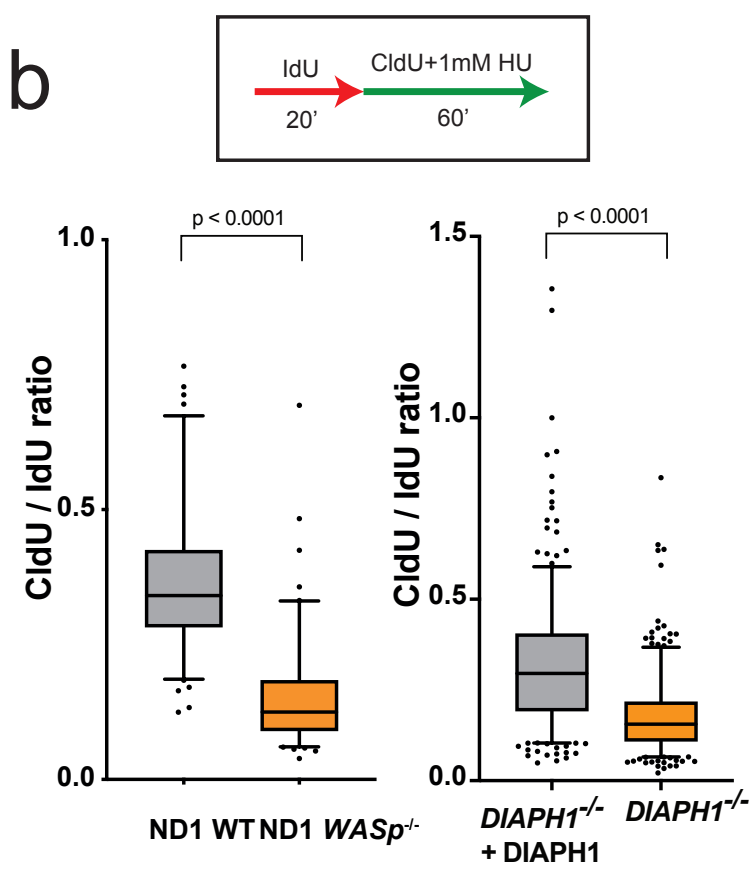

d

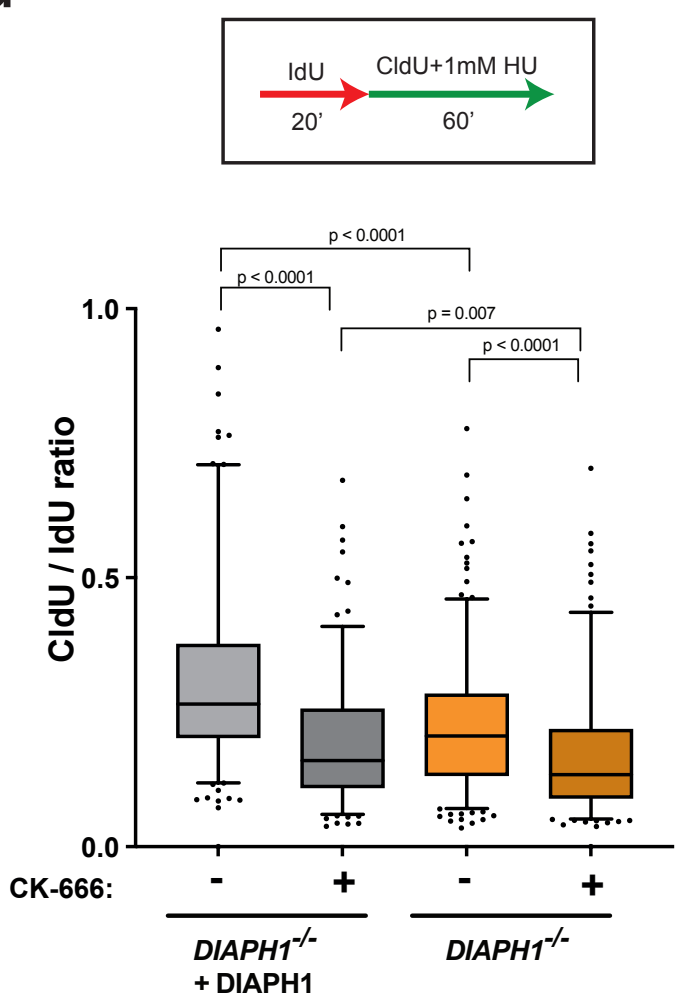

Fig.2 


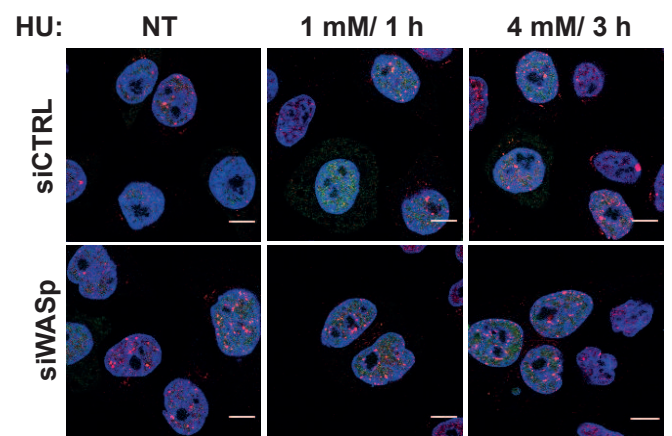

DAPI Cyclin A 53BP1

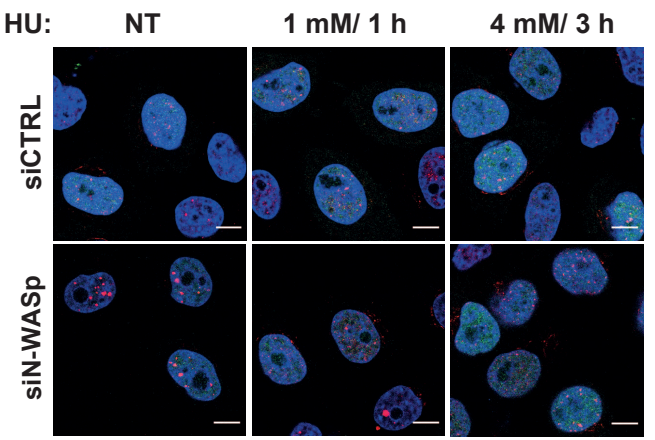

DAPI Cyclin A 53BP1

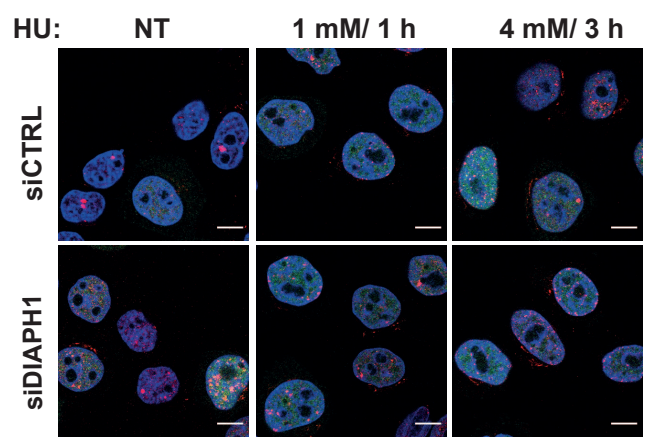

DAPI Cyclin A 53BP1
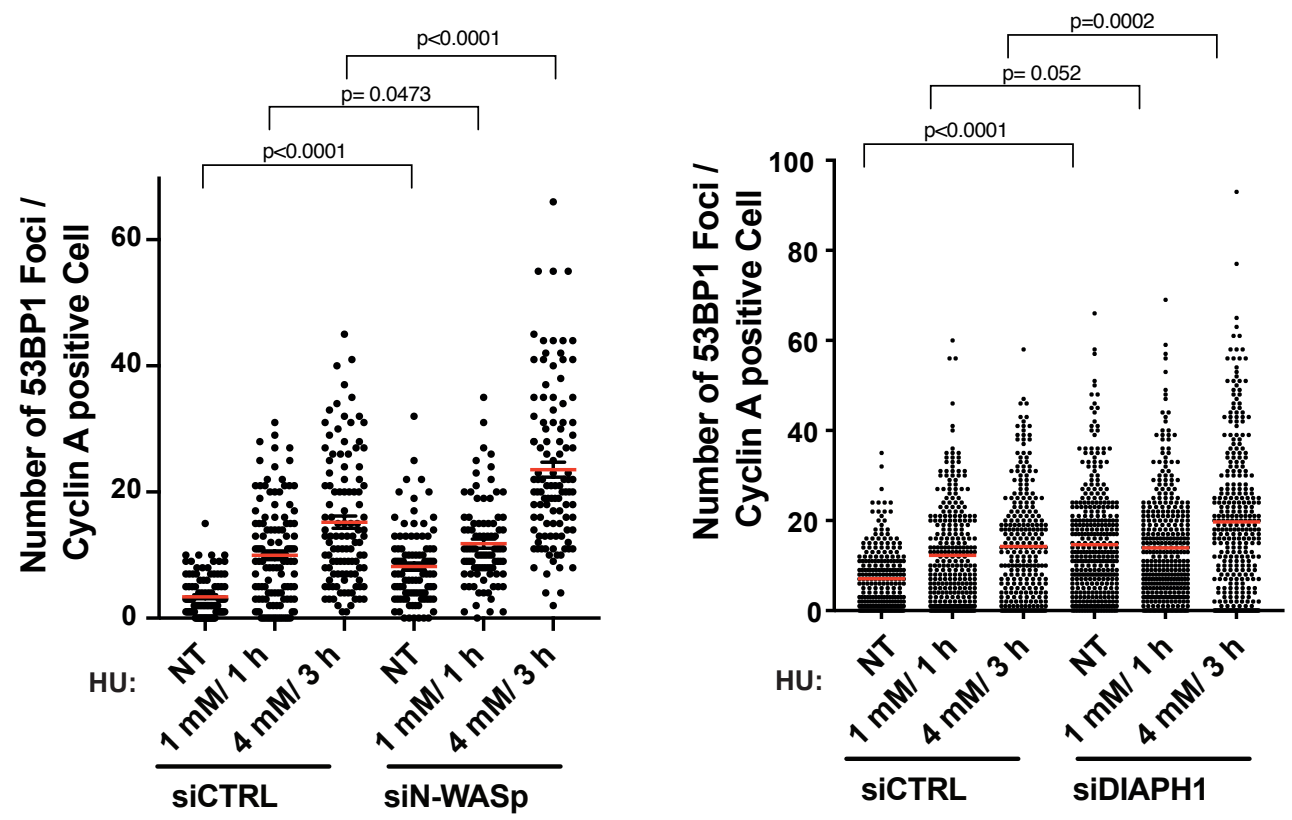

b
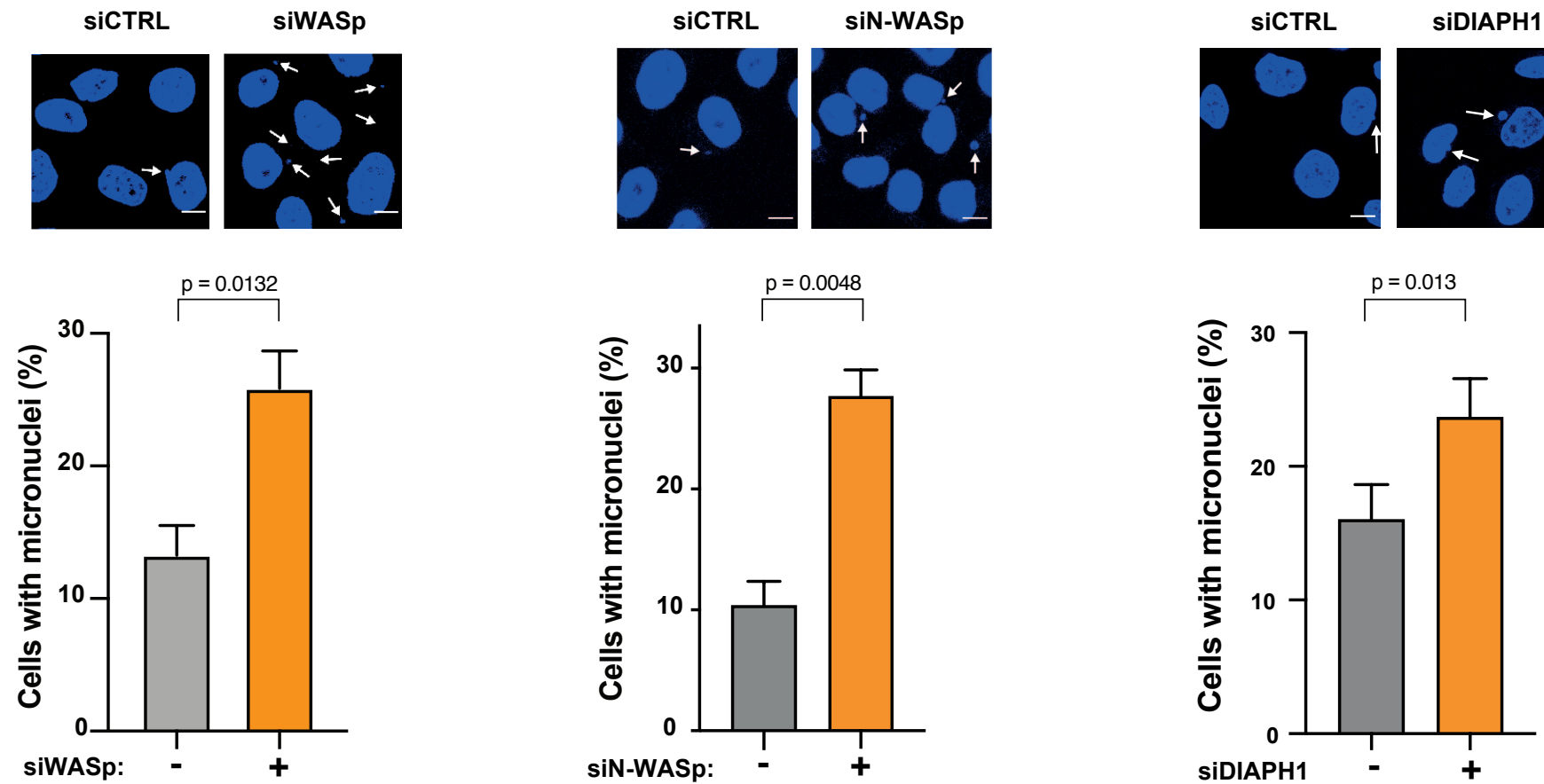

Fig. 3 
b

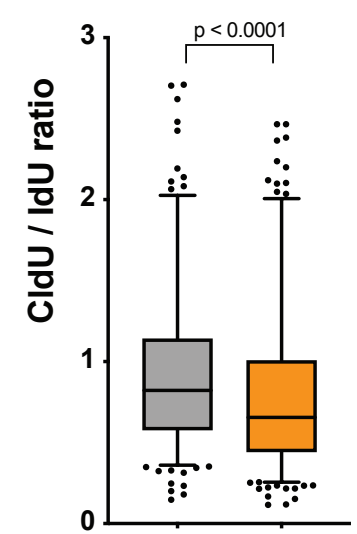

siWASp:

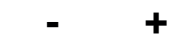

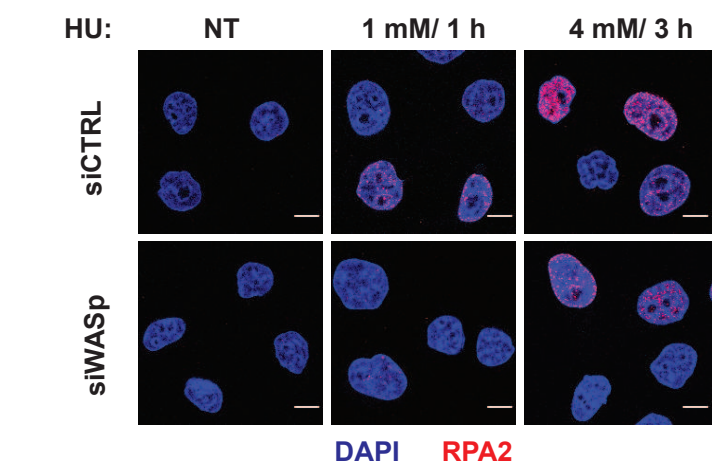

DAPI RPA2


DAPI RPA2




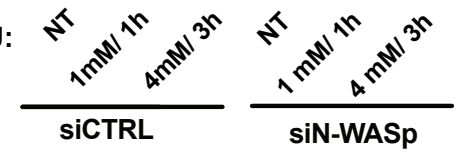

HU:

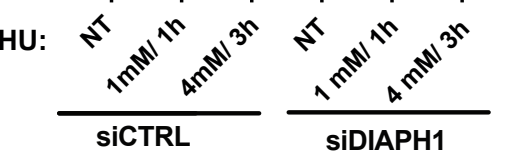

C

PLA: anti-RAD51 / anti-Biotin

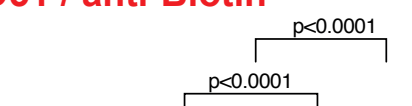

d

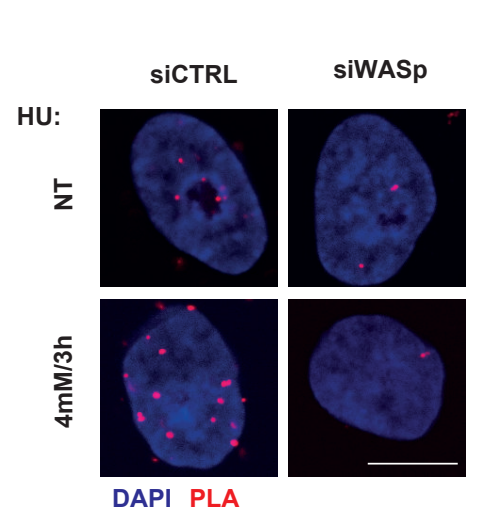

Fig.4

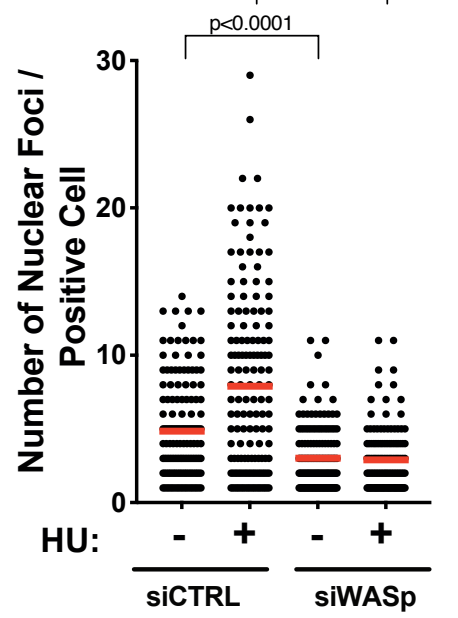

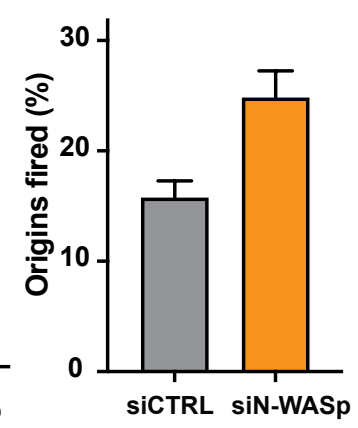

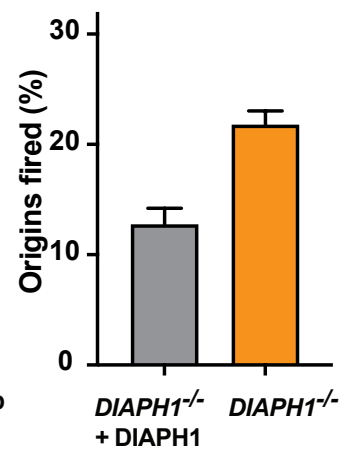



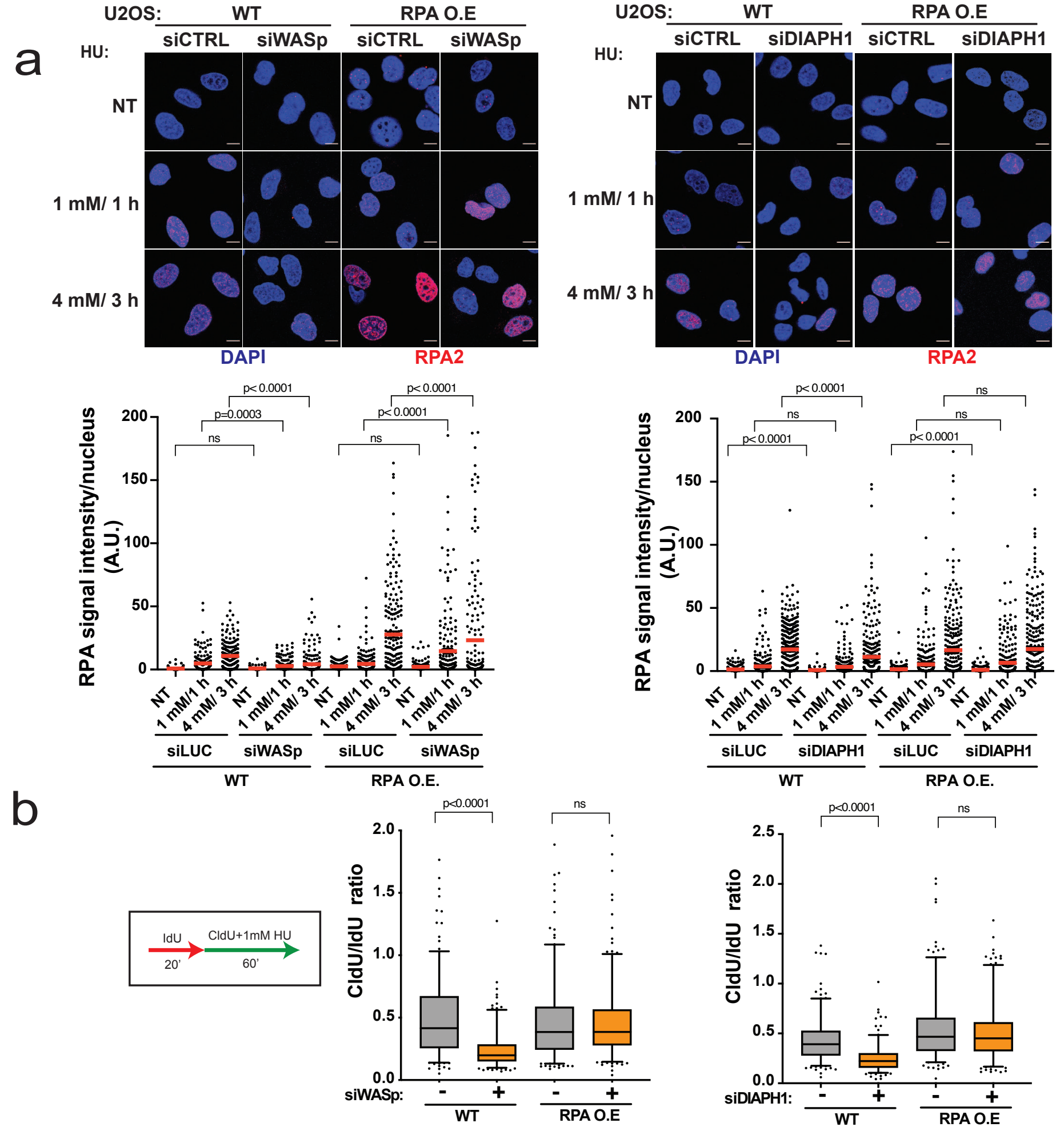

Fig. 5
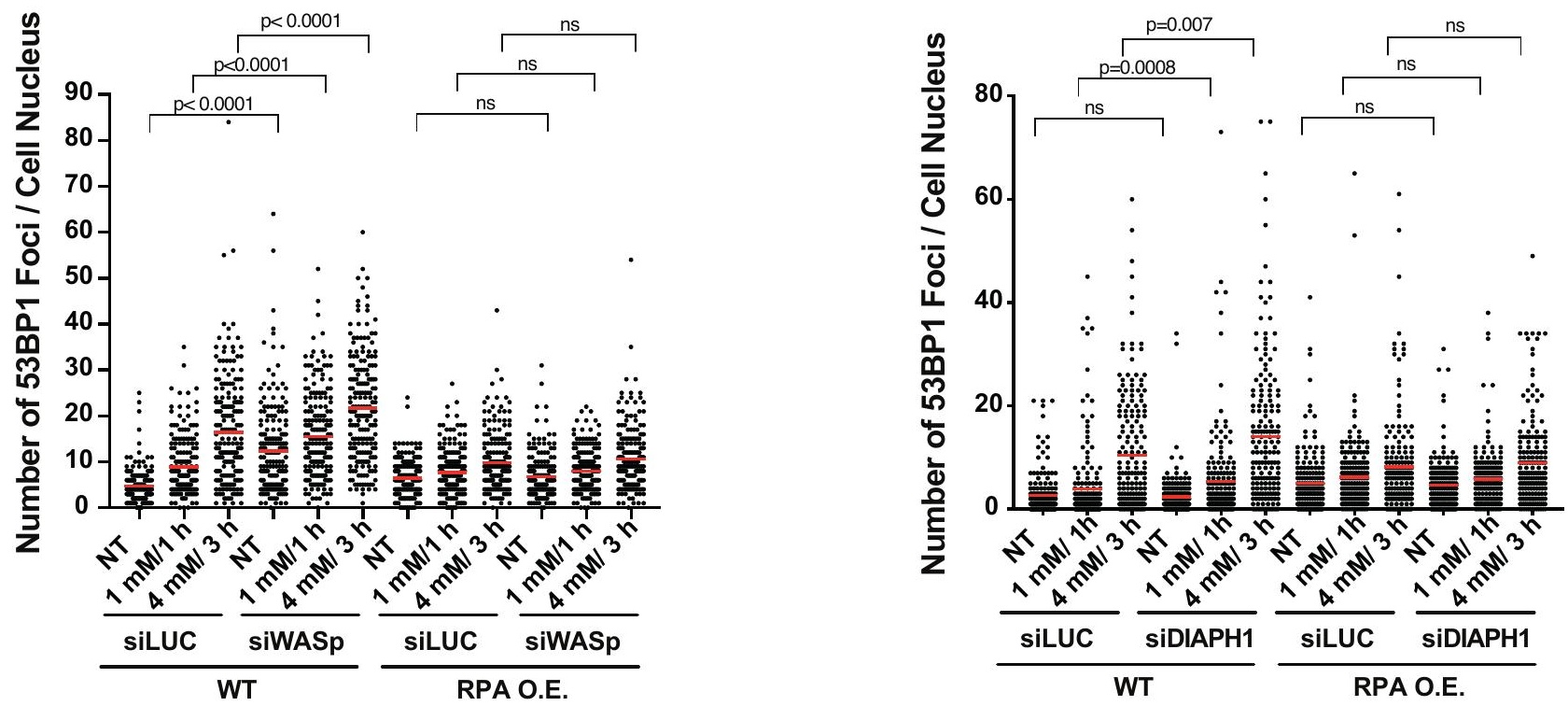


\section{Supplementary Files}

This is a list of supplementary files associated with this preprint. Click to download.

- Supplementaryinformation.pdf 